\title{
Stickstoffverbindungen von Elementen der dritten Hauptgruppe mit intra- und intermolekularen Donor-Akzeptor-Bindungen. I. Synthesen ${ }^{1)}$
}

\section{Michael Veith*, Holger Lange, Andreas Belo und Otmar Recktenwald}

Institut für Anorganische und Analytische Chemie der Technischen Universität Braunschweig, Pockelsstr. 4, D-3300 Braunschweig

Eingegangen am 13. April 1984

Die Wasserstoffatome im N,N'-Di-tert-butyl-Si,Si-dimethylsilandiamin (8) können durch Elemente der dritten Hauptgruppe, die organische Reste (Methyl, Phenyl) oder Chloratome als weitere Liganden tragen, substituiert werden. In den entstehenden Molekülen sind Atome vereinigt, die als Elektronenakzeptoren (Elemente der dritten Hauptgruppe) und als Elektronendonoren (Stickstoffatome) zusätzliche Bindungen untereinander eingehen können. Im einzelnen wurden nach unterschiedlichen Methoden synthetisiert: (a) Diazasilaelementetidine $\mathrm{Me}_{2} \mathrm{Si}(\mathrm{N} t \mathrm{Bu})_{2} \mathrm{El}-\mathrm{X}$ $(\mathrm{X}=\mathrm{Me}, \mathrm{El}=\mathrm{Al}(\mathbf{2 h})$, In (2d); $\mathrm{X}=\mathrm{Ph}, \mathrm{El}=\mathrm{Al}(\mathbf{2 e}))$, die allesamt als Koordinationsdimere vorliegen, (b) das durch Koordination von Pyridin am Aluminiumatom stabilisierte Diazasilaaluminetidin $\mathrm{Me}_{2} \mathrm{Si}(\mathrm{N} t \mathrm{Bu})_{2} \mathrm{Al}(\mathrm{Me}) \cdot \mathrm{NC}_{5} \mathrm{H}_{5}$ (3), (c) disubstituierte $N, N^{\prime}$-Di-tert-butyl-Si, Sidimethylsilandiamine des Typs $\mathrm{X}_{2} \mathrm{El}-\mathrm{N}(t \mathrm{Bu})-\mathrm{SiMe}_{2}-\mathrm{N}(t \mathrm{Bu})-\mathrm{ElX}_{2}(\mathrm{X}=\mathrm{Me}, \mathrm{El}=\mathrm{Al}(\mathbf{4} \mathbf{b})$, $\mathrm{Ga}(\mathbf{4 c})$, In (4d); $\mathrm{X}=\mathrm{Cl}, \mathrm{El}=\mathrm{Al}(\mathbf{4 e}))$, (d) einfach substituierte $N, N^{\prime}$-Di-tert-butyl-Si,Sidimethylsilandiamine des Typs $\mathrm{X}_{2} \mathrm{El}-\mathrm{N}(t \mathrm{Bu})-\mathrm{SiMe}_{2}-\mathrm{N}(t \mathrm{Bu})-\mathrm{H}(\mathrm{X}=\mathrm{Me}, \mathrm{El}=\mathrm{B}(\mathbf{5 a}), \mathrm{Al}$ (5b), Ga (5c), In (5d), Tl (5e); X = Cl, $\mathrm{El}=\mathrm{B}(5 \mathrm{f}), \mathrm{Al}(5 \mathrm{~g}), \mathrm{Ga}(5 \mathrm{~h}), \mathrm{In}(5 \mathrm{i}))$ und (e) hierzu verwandte Verbindungen der allgemeinen Formel $t \mathrm{Bu}-\mathrm{O}-\mathrm{SiMe}_{2}-\mathrm{N}(t \mathrm{Bu})-\mathrm{ElX}_{2}(\mathrm{X}=\mathrm{Me}$, $\mathrm{El}=\mathrm{Al}(\mathbf{6 a}) ; \mathrm{X}=\mathrm{Cl}, \mathrm{El}=\mathrm{Ga}(\mathbf{6 b}))$.

\section{Nitrogen Compounds of Elements of Main Group III with Intra- and Intermolecular Donor-Acceptor Bonds. - I. Syntheses 1)}

The hydrogen atoms in $N, N^{\prime}$-di-tert-butyl-Si, Si-dimethylsilanediamine (8) can be substituted by main group III elements, to which organic groups (methyl, phenyl) or chlorine atoms are bound. The resulting molecules combine atoms, which are electron acceptors (Group III elements) or electron donors (nitrogen atoms) and thus may cause additional bonds. In detail the following systems have been prepared by different methods: (a) diazasilaelementetidines $\mathrm{Me}_{2} \mathrm{Si}(\mathrm{N} t \mathrm{Bu})_{2}-$ $\mathrm{El}-\mathrm{X}(\mathrm{X}=\mathrm{Me}, \mathrm{El}=\mathrm{Al}(\mathbf{2 b}), \mathrm{In}(\mathbf{2 d}) ; \mathrm{X}=\mathrm{Ph}, \mathrm{El}=\mathrm{Al}(\mathbf{2 e}))$, which are dimerized by intermolecular coordination, (b) the diazasilaaluminetidine $\mathrm{Me}_{2} \mathrm{Si}(\mathrm{N} t \mathrm{Bu})_{2} \mathrm{Al}(\mathrm{Me}) \cdot \mathrm{NC}_{5} \mathrm{H}_{5}$ (3), which is stabilized at the aluminium atom by pyridine, (c) disubstituted $N, N^{\prime}$-di-tert-butyl-Si,Sidimethylsilanediamines $\mathrm{X}_{2} \mathrm{El}-\mathrm{N}(t \mathrm{Bu})-\mathrm{SiMe}_{2}-\mathrm{N}(t \mathrm{Bu})-\mathrm{ElX}_{2}(\mathrm{X}=\mathrm{Me}, \mathrm{El}=\mathrm{Al}(\mathbf{4 b}), \mathrm{Ga}(\mathbf{4 c})$, In (4d); $\mathrm{X}=\mathrm{Cl}, \mathrm{El}=\mathrm{Al}(\mathbf{4 e})$ ), (d) monosubstituted $N, N^{\prime}$-di-tert-butyl-Si,Si-dimethylsilanediamines $\mathrm{X}_{2} \mathrm{El}-\mathrm{N}(t \mathrm{Bu})-\mathrm{SiMe}_{2}-\mathrm{N}(t \mathrm{Bu})-\mathrm{H}(\mathrm{X}=\mathrm{Me}, \mathrm{El}=\mathrm{B}(\mathbf{5 a}), \mathrm{Al}(\mathbf{5} \mathbf{b}), \mathrm{Ga}(\mathbf{5 c}), \mathrm{In}(5 \mathrm{~d})$, $\mathrm{Tl}(5 \mathrm{e}) ; \mathrm{X}=\mathrm{Cl}, \mathrm{El}=\mathrm{B}(5 \mathbf{f}), \mathrm{Al}(5 \mathrm{~g}), \mathrm{Ga}(5 \mathbf{h})$, In $(5 \mathrm{i}))$, and (e) related compounds of the formula $t \mathrm{Bu}-\mathrm{O}-\mathrm{SiMe}_{2}-\mathrm{N}(t \mathrm{Bu})-\mathrm{EIX}_{2}(\mathrm{X}=\mathrm{Me}, \mathrm{El}=\mathrm{Al}(6 \mathbf{a}) ; \mathrm{X}=\mathrm{Cl}, \mathrm{El}=\mathrm{Ga}(6 \mathbf{b}))$.

Wie wir in einer Reihe von Arbeiten zeigen konnten (Übersicht siehe Lit. ${ }^{2}$ ), fungieren die cyclischen Diazagermylene, -stannylene und -plumbylene 1a, 1 b und $1 \mathrm{c}$ als wertvolle Synthone für anorganische und metallorganische Verbindungen des zweiwertigen Germaniums, Zinns und Bleis.

(C) VCH Verlagsgesellschaft mbH, D-6940 Weinheim, 1985

$0009-2940 / 85 / 0404-1600 \$ 02.50 / 0$ 
Als Besonderheit dieser Verbindungen kann das gleichzeitige Vorliegen von Lewis-sauren (niederwertiges Element) und Lewis-basischen Zentren (Stickstoffatome) angesehen werden, die je nach Reaktionspartner zu einer Fülle unterschiedlicher Reaktionen führen ${ }^{3)}$. Auch strukturchemisch sind diese kleinen Ringsysteme interessant; je nach Aggregatszustand bzw. Modifikation findet man bei $1 \mathrm{~b}$ z. B. monomere oder dimere Moleküle, die über intermolekulare Lewis-Säure-BaseWechselwirkungen zustande kommen ${ }^{4)}$.

Um die Ringsysteme 1a-c in einen größeren Zusammenhang mit anderen anorganischen Ringen zu stellen, haben wir versucht, das niederwertige Element in 1 durch andere, typische LewisSäuren zu ersetzen. Insbesondere erschien es uns sinnvoll, Elemente der dritten Hauptgruppe zu berücksichtigen, da nach Einbau in das vorgegebene Ringsystem die formale Außenelektronenzahl an den Elementen $\mathrm{El}$ in $\mathbf{1}$ und $\mathbf{2}$ identisch sein sollte.

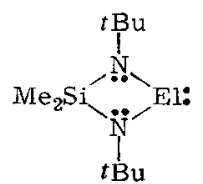

$$
\begin{array}{l|lll}
\mathbf{1} & \mathbf{a} & \mathbf{b} & \mathbf{c} \\
\hline \mathrm{El} & \mathrm{Ge} & \mathrm{Sn} & \mathrm{Pb}
\end{array}
$$

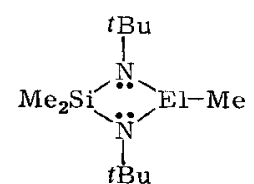

\begin{tabular}{l|llll}
$\mathbf{2}$ & a & b & c & d \\
\hline El & B & Al & Ga & In
\end{tabular}

Ein ganz allgemeines Ziel dieser Untersuchungen ist, etwas über die Struktur und Reaktivität von kleinen Ringen zu lernen, die wie 1 und $\mathbf{2}$ gegensätzliche elektrophile und nucleophile Zentren enthalten. Während die Borverbindung $2 \mathbf{a}$ bereits beschrieben war ${ }^{5)}$, mußten die Ringe $\mathbf{2 b}-\mathbf{d}$ neu synthetisiert werden. Bei den Darstellungsversuchen zu 2 konnten wir eine Reihe von Vorstufen bzw. Zwischenstufen (3, $\mathbf{4}$ und 5) isolieren, die mit Ausnahme von $\mathbf{3}$ auf den ersten Blick acyclisch erscheinen, aber auf Grund der im Molekül vorhandenen Elektronen-Donoren und -Acceptoren zu intramolekularen Ringschlüssen neigen sollten (siche auch Lit. 27, 28)). Strukturelle Untersuchungen zu diesem Phänomen sowie die vollständige Strukturbestimmung eines Vertreters von 2 haben wir in gesonderten Veröffentlichungen zusammengefaßt ${ }^{1)}$. Ein 5 sehr ähnliches System 6, das durch formalen Austausch der (NH)-Gruppe durch Sauerstoff zustandekommt, wird ebenfalls erstmals beschrieben.<smiles>CCCCN1N([Al](C)(C)CCCC)[Si]1(C)C</smiles>

3<smiles>[Y10]N([14CH3])[SiH2]N([13CH3])[14CH3]</smiles>

$\mathrm{El}=\mathrm{Al} ; \mathrm{Ga}, \mathrm{In}$ $\mathrm{X}=\mathrm{Me}, \mathrm{Cl}$

4<smiles>[Y10]N([14CH3])[Si](C)(C)NCCCC</smiles>

$\mathrm{El}=\mathrm{B}, \mathrm{Al}, \mathrm{Ga}, \mathrm{In}, \mathrm{Tl}$ $\mathrm{X}=\mathrm{Me}, \mathrm{Cl}$

5<smiles>CCCCO[Si](C)N(CCC)CCCC</smiles>

$\mathrm{El}=\mathrm{Al}, \mathrm{Ga}$ $\mathrm{X}=\mathrm{Me}, \mathrm{Cl}$

6

Reaktionen zum Aufbau der Atomverknüpfung $-\mathbf{E} \mathbf{I}-\mathbf{N}-\mathbf{S i}-\mathbf{N}-\mathbf{E}-\stackrel{\mid}{\mid}-\mathbf{\text { (4) }}$

Wie vorstehend angedeutet, sind die Diazasilaelementetidine 2 meist nur über Vorstufen zugänglich. Eine dieser Ausgangsverbindungen, ein durch DimethylelementGruppen substituiertes Silandiamin 4, läßt sich nach Reaktionsschema (1) über verschiedene Wege darstellen.

Chem. Ber. 118 (1985) 
(1a)

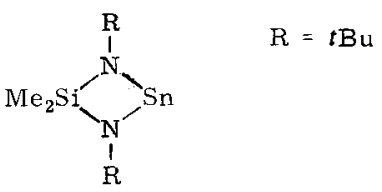

lb<smiles>[R]N([14CH3])[SiH]([14CH3])N([R])[34CH3]</smiles>

5 (1b)<smiles>[R]N1[SiH](C)N([AsH3])[SiH]1C</smiles>

7
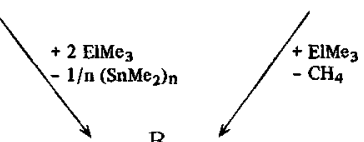

Wie aus Gl. (1a) ersichtlich, entstehen die Verbindungen 4 durch die Umsetzung des cyclischen Bis(amino)stannylens $1 \mathrm{~b}$ mit Trimethylelement-Verbindungen der dritten Hauptgruppe. Die Ausbeuten an 4 b, c,d betragen $85-90 \%$, wobei bei der Isolierung der Verbindungen die leichte Trennbarkeit von $\mathbf{4 b}, \mathbf{c}, \mathbf{d}$ von polymerem Dimethylstannan durch Sublimation im Vakuum hervorzuheben ist. Die Reaktion findet sowohl bei Raumtemperatur als auch bei $-78^{\circ} \mathrm{C}$ statt. Letzterer Punkt ist insofern bemerkenswert, als hierdurch die Möglichkeit besteht, das intermediär auftretende Dimethylstannylen, das sich dann zum Polymeren stabilisiert, unter schonenden Bedingungen darzustellen (vgl. Lit. ${ }^{6}$ ). Die Reaktion (1a) kann als klassische Metallaustauschreaktion betrachtet werden, wobei wahrscheinlich die basischen Stickstoffatome in $\mathbf{1 b}$ als Angriffspunkte für $\mathrm{Al}_{2} \mathrm{Me}_{6}, \mathrm{GaMe}_{3}$ und $\mathrm{InMe}_{3}$ dienen. Einen Angriff der Lewis-Säuren am einsamen Elektronenpaar des Zinnatoms in $\mathbf{1 b}$ - eine Möglichkeit über die in der Literatur spekuliert wird ${ }^{7)}$ - halten wir auf Grund der ganz ähnlich verlaufenden Reaktion (1b) für wenig wahrscheinlich. Hierbei reagiert die Verbindung 7, die aus dem Dilithiumsalz des Bis(amino)dimethylsilans 8 und Dichlordimethylstannan unter Lithiumchloridabspaltung entsteht, mit Trimethylelement-Verbindungen unter Übertragung von zwei Methylgruppen auf das Zinnatom und der Bildung von 4. Im Unterschied zu $1 \mathrm{~b}$ verfügt das vierwertige Zinnatom in 7 über kein freies Elektronenpaar!

Die Darstellung von 4 aus den Verbindungen 5, die über eine Reihe von Synthesewegen zugänglich sind (siehe nächstes Kapitel), und Trimethylelement-Verbindungen unter Methanabspaltung erfordert Temperaturen von über $60^{\circ} \mathrm{C}$. Befriedigende Ausbeuten werden allerdings nur bei der Aluminium- und Indiumverbindung registriert, während beim Galliumderivat bis $110^{\circ} \mathrm{C}$ keine Methanabspaltung beobachtet wird. Wie ein Vergleich mit dem Reaktionsschema (3) zeigt, kann man 4 auch direkt aus 8 und dem Trimethylelement ohne Isolierung von 5 darstellen.

Reaktion (1d) ist als klassische Reaktion zum Aufbau von Amiden wohlbekannt ${ }^{8)}$, liefert aber im Fall der Synthesen von $4 \mathbf{b , c}, \mathbf{d}$ die niedrigsten Ausbeuten. Es sei noch darauf hingewiesen, daß an Stelle von $\mathbf{1} \mathbf{b}$ die homologen Verbindungen $1 \mathbf{a}$ und $1 \mathbf{c}$ zur 
Darstellung von $\mathbf{4 b , c , d}$ in Reaktion (1a) verwendet werden können, wobei die Ausbeuten an den Zielmolekülen vergleichbar sind (siehe Experimenteller Teil).

Bei den Verbindungen $\mathbf{4 b , c}$ und $\mathbf{d}$ handelt es sich um farblose, leicht verformbare Feststoffe, die, wie an einem Kristall von $\mathbf{4 b}$ nachgewiesen (Tab. 1), der „plastischen Phase" angehören. Die ${ }^{1} \mathrm{H}-\mathrm{NMR}$-Spektren bei Raumtemperatur bestehen aus jeweils drei Singuletts, die für die Resonanzen der Methylgruppen am Kohlenstoff-, am Silicium- und Aluminium- bzw. Gallium- bzw. Indiumatom stehen. Alle Verbindungen liegen in Benzol monomer vor. Es sei hier bereits angedeutet, daß $\mathbf{4 b}, \mathbf{c}$ und $\mathbf{d}$ keine offenkettigen Systeme darstellen, sondern in Lösung komplexe Bindungsfluktuationen unter Einbeziehung von Donor-Acceptor- und Zwei-Elektronen-Drei-Zentren-Bindungen ablaufen ${ }^{1)}$.

Tab. 1. Die „plastische Phase“ ${ }^{\text {26) }}$ von 4 b nach Einkristall-Aufnahmen

\begin{tabular}{ll} 
Kristallsystem: kubisch & $V=989.8 \cdot 10^{6} \mathrm{pm}^{3}$ \\
Raumgruppe: & $Z=2($ Punktsymmetrie des Moleküls: \\
$I m 3 m$ bzw. $I \overline{4} 3 m$ & $\begin{array}{l}m 3 m \text { bzw. } \overline{43 m}) \\
a=996.6(5) \mathrm{pm}\end{array}$ \\
\hline
\end{tabular}

Die zweifach substituierten Silandiamine 4 lassen sich ohne Aufbrechen der Siliciumoder Element-Stickstoffbindung chemisch modifizieren. So ist es uns entsprechend G1. (2) gelungen, in $\mathbf{4 b}$ durch Umsetzung mit 4 Äquivalenten Chlortrimethylstannan die Methylgruppen am Aluminiumatom durch Chloratome zu ersetzen.

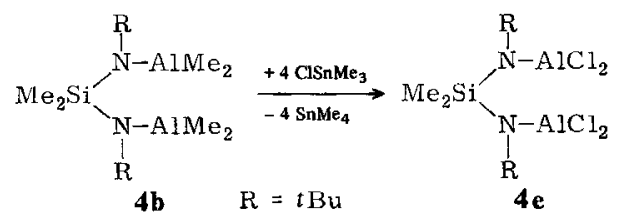

Neben $4 \mathbf{e}$ entsteht bei dieser Reaktion Tetramethylstannan, das sich einfach vom Produkt abtrennen läßt. Ein mit Dimethylboryl-Gruppen substituiertes Silandiamin $\mathrm{Me}_{2} \mathrm{~B}-\mathrm{N}(t \mathrm{Bu})-\mathrm{SiMe}_{2}-\mathrm{N}(t \mathrm{Bu})-\mathrm{BMe}_{2}(\mathbf{4} \mathrm{a})$ ist nach Nöth et al. ${ }^{5)}$ nicht stabil und zerfällt bei Raumtemperatur spontan in $\mathbf{2 a}$ und Trimethylboran.

\section{Reaktionen zum Aufbau der Atomverknüpfung $-\frac{1}{\mathbf{E l}}-\mathbf{N}-\mathbf{S} i-\frac{1}{\mathrm{~N}}-\mathbf{H}(5)$}

Die Substitution eines Wasserstoffatoms im $N, N^{\prime}$-Di-tert-butyl-Si, Si-dimethylsilandiamin (8) durch eine Lewis-saure Gruppe wie ElMe ${ }_{2}$ ist uns für alle Elemente El der dritten Hauptgruppe gelungen. Die verschiedenen Synthesewege, die zu den Verbindungen 5a-e führen, sind im Reaktionsschema (3) zusammengefaßt.

Die am allgemeinsten anwendbaren Darstellungsmethoden sind in den Gln. (3a) und (3b) angegeben. Die hierzu benötigte Zinnverbindung 9 ist in zwei Schritten aus dem Silandiamin 8 gemäß GI. (4) zugänglich, wobei zur Vermeidung von Nebenreaktionen 8 im Überschuß vorgelegt wird.

Bei den Reaktionen ( $3 a$ ) und ( $3 b$ ) werden jeweils die reaktiven $\mathrm{Sn}-\mathrm{N}$-Bindungen zur Synthese von 5 ausgenützt, wobei sicher auch die hohe Bildungstendenz von Tetramethylstannan bzw. Bromtrimethylstannan eine Rolle spielt. Die Ausbeuten an 5 sind 
(3b)<smiles>[R]N([SiH3])[Si]([R])(C)N([R])[AsH3]</smiles>

9

(3c)<smiles>[R]N[Si](C)(C)N[R]</smiles>

8
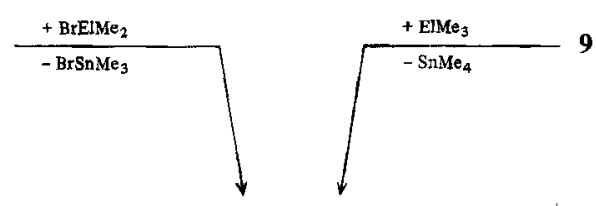

9
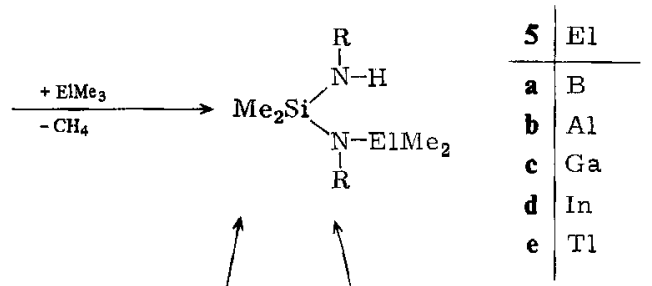

$R=t B u$

(3d)<smiles>[R]N(C)[SiH](C)N([R])[13CH3]</smiles>

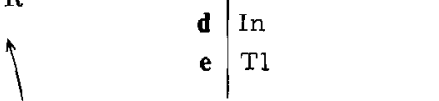

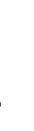

4<smiles>[R]N[Si](C)(C)N[Y]([R])[H]</smiles>

8<smiles>[R]N([AlH])[Al]([R1])[Sn](C)(C)C</smiles>

9

$$
\mathrm{R}=t \mathrm{Bu}
$$

nach diesen Methoden nahezu quantitativ. Die Abspaltung von Methan aus einer äquimolaren Mischung von 8 und Trimethylelement (Gl. (3c)) ist nur im Fall von Trimethylaluminium und Trimethylgallium zu empfehlen und nicht ganz unproblematisch, da bei zu hohen Reaktionstemperaturen die zweifach substituierten Silandiamine 4 neben 5 entstehen können. Reaktion (3d) ist eine Umaminierung, bei der Substituentenaustausch zwischen den Silandiaminen 8 und $\mathbf{4}$ stattfindet, bis schließlich nur noch 5 vorliegt. Letztere Methode haben wir nur (ebenso wie (3e)) für den Fall der Aluminiumverbindung experimentell verifiziert; sie scheint uns aber auch für andere Elementverbindungen möglich. Bei (3e) schließlich wird an Stelle von 8 Diethylamin als „Umaminierungsmittel" verwendet.

Die Verbindungen 5a,c - e sind farblose Flüssigkeiten bzw. Feststoffe (5b), die sich in unpolaren Lösungsmitteln wie Benzol oder Cyclohexan monomer auflösen. Die meisten dieser Moleküle lassen sich unzersetzt destillieren bzw. sublimieren und sind massenspektroskopisch einfach über den Molekülpeak nachweisbar (Ausnahme: 5e). Die ${ }^{1} \mathrm{H}-\mathrm{NMR}-S p e k t r e n$ von 5a,c,d und e in Benzol bei Raumtemperatur bestehen aus je einem Resonanzsignal für die $\mathrm{Me}_{2} \mathrm{Si}$ - und Dimethylelement-Gruppen sowie aus zwei Signalen für die chemisch unterschiedlichen tert-Butylgruppen. Das ${ }^{1} \mathrm{H}$-NMRSpektrum von $\mathbf{5 b}$ unterscheidet sich hiervon dadurch, daß sowohl das Signal der $\mathrm{Me}_{2} \mathrm{Si}$ - als auch der $\mathrm{Me}_{2} \mathrm{Al}$-Gruppe in zwei Singuletts aufgespalten ist. Eine weitergehende Untersuchung dieser Systeme bei verschiedenen Temperaturen zeigt, daß die 
acyclische Form von 5 mit einer Ringstruktur in Konkurrenz steht, bei der das Stickstoffatom der N(H)R-Gruppe das Lewis-saure Element koordiniert ${ }^{1)}$.

Analog zu Gl. (2) lassen sich auch in 5b gemäß Gl. (5) die Methylgruppen am Aluminiumatom durch Chloratome ersetzen.<smiles>[R]N([14CH3])[SiH2]N([R])[AlH2]</smiles>

Eine andere Methode, Elementdichloride in das System 5 einzuführen, besteht in der Reaktion (6a), die wir zur Darstellung von $5 \mathrm{~h}$ und $\mathbf{i}$ benutzt haben, oder in (6b), die zur Borverbindung $5 \mathrm{f}$ führt. Um Nebenreaktionen zu vermeiden (Bildung von 4), muß bei (6a) das Silandiamin $8 \mathrm{im}$ Überschuß eingesetzt werden.<smiles>[R]N[SiH]([R1])N[R]</smiles>

8

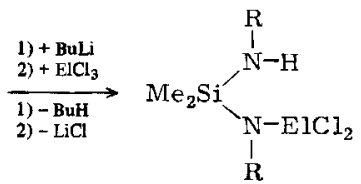

5h: $\mathrm{El}=\mathrm{Ga}$

5i : $\mathrm{E} 1=$ In

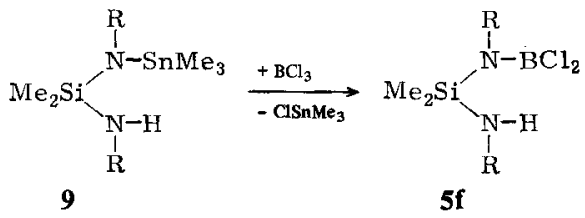

$\mathrm{R}=t \mathrm{Bu}$

Die Verbindungen $5 \mathrm{~g}-\mathbf{i}$ sind farblose Feststoffe, während $\mathbf{5 f}$ bei Normalbedingungen flüssig ist. In den ${ }^{1} \mathrm{H}-\mathrm{NMR}$-Spektren der in Benzol gelösten Verbindungen $\mathbf{5} \mathbf{g}$ und $\mathbf{h}$ treten neben den zwei Resonanzlinien für die tert-Butylgruppen zwei Linien für die $\mathrm{Me}_{2} \mathrm{Si}$-Gruppe auf; dahingegen besitzen die Spektren von $\mathbf{5 f}$ und $\mathbf{i}$ im MethylsilylBereich nur jeweils ein Resonanzsignal. Auch hier liegen ähnliche Strukturverhältnisse wie bei $5 \mathbf{a}-\mathbf{e}$ vor $^{1)}$.

Die mit den Molekülen 5 verwandten Amide 6, die sich von ersteren durch den Ersatz der Atomgruppe $\mathrm{NH}$ durch das nach Grimm $^{9}$ ) isostere Atom $\mathrm{O}$ unterscheiden, lassen sich aus den Amiden 10 nach Gl. (7) bzw. (8) in guten Ausbeuten darstellen.

$\mathbf{6 a}$ und $\mathbf{b}$ sind in Lösung (Benzol) und in der Gasphase (Massenspektrum) monomer und liegen bei Raumtemperatur als farblose Flüssigkeit (6a) bzw. als farblose Kristalle (6b) vor. In den ${ }^{1} \mathrm{H}-\mathrm{NMR}-S p e k t r e n$ findet man 4 Resonanzsignale für $6 \mathbf{a}$ bzw. 3 für $\mathbf{6 b}$, entsprechend den chemisch unterschiedlichen Methylgruppen. Inwieweit wie bei 5 auch bei 6a und b Ringstrukturen zu diskutieren sind, die aus der intramolekularen Koordination des Sauerstoffatoms zum Aluminium- bzw. Galliumatom hervorgehen, ist ohne 
Experimente schwierig zu beantworten. Wir halten solche Strukturen in Anbetracht der Ergebnisse bei $5^{1)}$ für wahrscheinlich.<smiles>[R]N[Si](C)(C)O[R]</smiles>

10

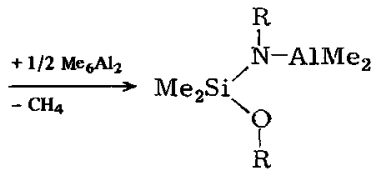

6a

$$
\mathrm{R}=t \mathrm{Bu}
$$<smiles>[R]O[Si](=[As])N([R])[14CH3]</smiles>

$6 \mathbf{b}$

\section{1,3-Diaza-2-sila-4-elementetidine mit Aluminium oder Indium als Ring- komponente}

Die den Ringverbindungen 1 entsprechenden Cyclen $\mathbf{2} \mathbf{b}, \mathbf{c}$ und $\mathbf{d}$, mit Elementen der dritten Hauptgruppe als Ringbausteinen, lassen sich gemäß Reaktionsschema (9) prinzipiell nach fünf verschiedenen Methoden darstellen.

(9b)

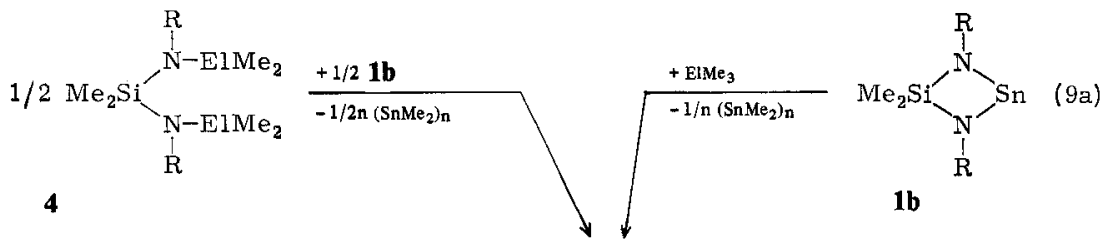

(9c)
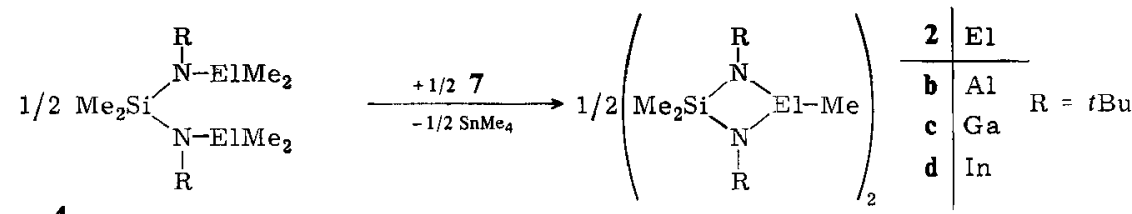

(9d)<smiles>[R]N[Si](C)(C)N([R])C(C)(C)C</smiles>

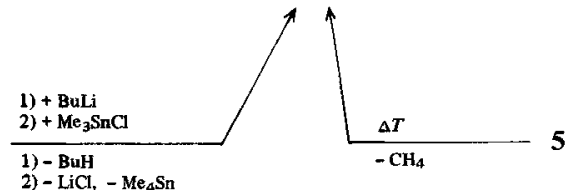

5

Die Reaktionen (9a), (9b) und (9c) ähneln sich insofern, als in allen drei Fällen eine Stickstoff-Zinn-Bindung gelöst wird, während dafür eine Element-Stickstoff-Bindung geschlossen wird. Wie bei den bereits beschriebenen Gleichungen (1a) und (1b) sind Methylzinn-Verbindungen die neben 2 entstehenden Produkte. Die Ausbeuten an Aluminiumderivat $\mathbf{2 b}$ nach $(9 \mathbf{a}-\mathbf{c})$ sind sehr hoch, diejenigen des Indiumderivates $2 \mathbf{d}$ 
niedriger $(40 \%)$. Die Galliumverbindung $2 \mathrm{c}$ ist weder nach diesen Methoden, noch nach (9d) und (9e) zugänglich; der Grund hierfür liegt in bisher noch untrennbaren Substanzgemischen, also in der Aufarbeitung. Wie wir im Fall der Darstellung von 2 b fanden, stehen die Reaktionen (9a) und (9b) in direkter Beziehung zueinander. Setzt man zu einer Lösung von $\mathbf{1 b}$ in Toluol tropfenweise $\mathrm{Al}_{2} \mathrm{Me}_{6}$ hinzu, so entsteht bei Raumtemperatur $2 \mathrm{~b}$ in $100 \%$ Ausbeute, bei $-78^{\circ} \mathrm{C}$ allerdings nur zu $50 \%$. In letzterem Fall ist $\mathbf{4 b}$ das Nebenprodukt. Ändert man die Reihenfolge der Zugabe, indem man $\mathrm{Al}_{2} \mathrm{Me}_{6}$ vorlegt und $1 \mathrm{~b}$ zutropft, so findet man jetzt bei Raumtemperatur ein Verhältnis von $50 \% 2 \mathrm{~b}$ neben $50 \%$ 4b und bei $-78^{\circ} \mathrm{C} 43 \% 2 \mathrm{~b}$ neben $57 \% 4 \mathrm{~b}$ (aus den ${ }^{1} \mathrm{H}-\mathrm{NMR}$-Spektren der Reaktionslösung). Diese Ergebnisse lassen erahnen, daß möglicherweise mehrere Konkurrenzreaktionen nebeneinander ablaufen, wie in Gl. (10) angedeutet, bzw. 4b eine Zwischenstufe in Reaktion (9a) darstellt. Kinetische Untersuchungen könnten hierzu weitere Klärung bringen.
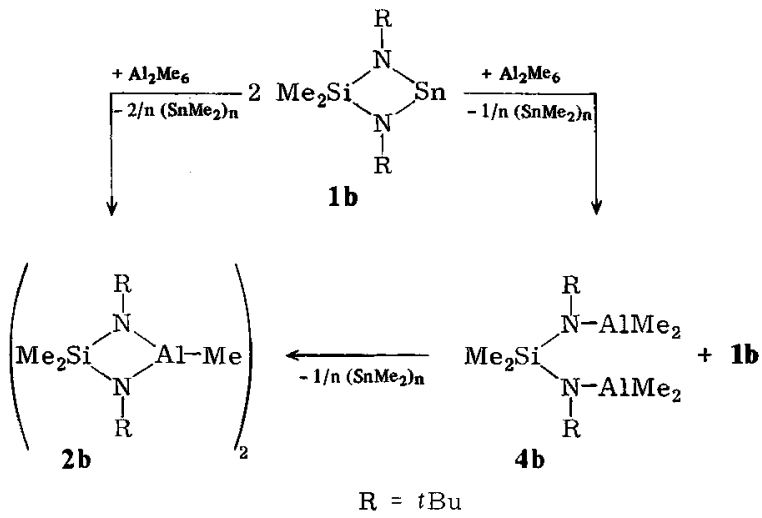

Von allen Reaktionen im Gleichungssystem (9) führt die Methode (9d) zu den höchsten Ausbeuten an 2. In einem ersten Schritt wird das Wasserstoffatom in $\mathbf{5}$ durch ein Lithiumatom ausgetauscht, dem dann die Substitution durch eine TrimethylstannylGruppe folgen sollte. Die Zwischenstufe $\mathrm{Me}_{2} \mathrm{El}-\mathrm{N}(\mathrm{R})-\mathrm{SiMe}_{2}-\mathrm{N}(\mathrm{R})-\mathrm{SnMe}_{3}$ kann man nicht isolieren, da sie unter den Reaktionsbedingungen unter Abspaltung von Tetramethylstannan zum Cyclus 2 kondensiert. Die Methode (9e) ist schließlich nur für die Darstellung von $\mathbf{2 b}$ geeignet, wobei die Reaktionstemperatur zur Abspaltung von Methan aus $5 \mathrm{~b} 130^{\circ} \mathrm{C}$ beträgt. Die analoge Reaktion mit $5 \mathrm{c}$ und $5 \mathrm{~d}$ ist ohne Zersetzung der Edukte nicht möglich.

$2 \mathbf{b}$ und $\mathbf{d}$ sind kristallin, lösen sich als Dimere in Benzol und erscheinen im Massenspektrum als dimer (2b) oder monomer (2d). Das ${ }^{1} \mathrm{H}-\mathrm{NMR}-$ Spektrum von $\mathbf{2 b}$ besteht aus insgesamt 5 Absorptionen: jeweils doppelte Resonanzsignale für die tert-Butyl- und Dimethylsilyl-Gruppen sowie 1 Signal für die Methylaluminium-Gruppe werden beobachtet. Im Unterschied hierzu besitzt das Spektrum von $\mathbf{2 d}$ nur drei Signale: die Resonanzen im Dimethylsilyl- bzw. tert-Butyl-Bereich entarten zu zwei Singuletts. Letztere Erscheinung hat ihre Ursache in Bindungsfluktuationen innerhalb des Moleküls 2d, die bei Raumtemperatur im Gegensatz zu $2 \mathbf{b}$ schnell gegenüber der ${ }^{1} \mathrm{H}-\mathrm{NMR}$-Zeitskala ablaufen ${ }^{1)}$. In Anlehnung an die Verhältnisse in kristallinem $1 \mathbf{b}^{10}$ ) ergibt sich aus den 
NMR-Spektren von $\mathbf{2 b}$ und d der Strukturvorschlag I, der durch Röntgenstrukturanalyse im Fall von $\mathbf{2 d}$ belegt werden kann ${ }^{1)}$.

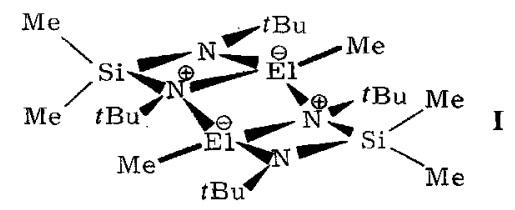

Grundsätzlich bleibt festzuhalten, daß die Vierringe $\mathbf{2 b}$ und $\mathbf{d}$ im Unterschied zu $\mathbf{2 a}$ $(E l=B)^{5)}$ und jenen des Formeltyps 1 in unpolaren organischen Lösungsmitteln nicht monomer vorliegen. Man kann daraus folgern, daß die Lewis-Aciditäten von Al(III) und In(III) in 2 generell größer sind als die von $\mathrm{Ge}$ (II), $\mathrm{Sn}$ (II) und $\mathrm{Pb}$ (II) in 1 bzw. von $\mathrm{B}(\mathrm{III})$ in $2 \mathbf{a}$. Auch die formale Substitution der Methylgruppe am Aluminiumatom in 2b durch eine Phenylgruppe (2e, Darstellung entsprechend Gl. (11)) ändert nichts an der Koordinationszahl 4 des Aluminiumatoms.

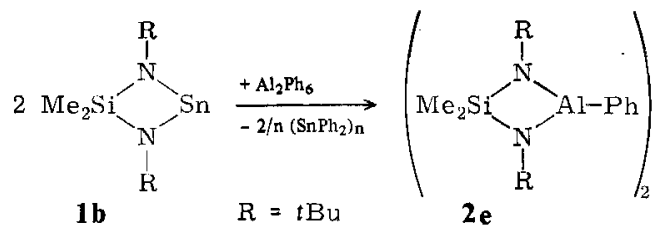

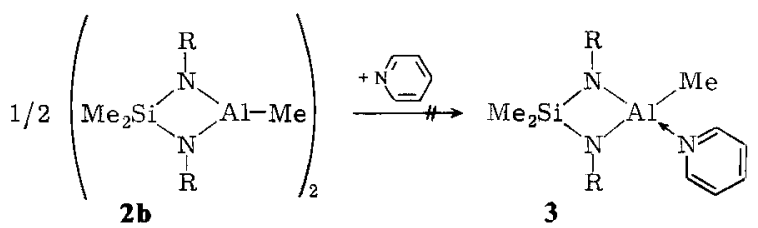<smiles>[R]N1[AlH]N([R])[AlH]1</smiles>

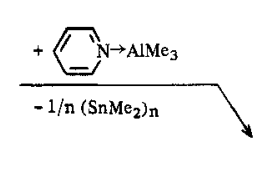

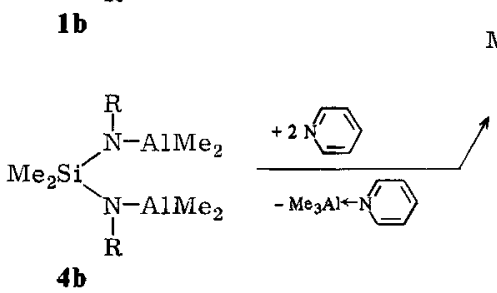<smiles>[R]N1[SiH](C)N([R])[Y]1(n1cccc1)n1cccc1</smiles>

Koordinationsdimere, wie wir sie bei $\mathbf{2} \mathbf{b}, \mathbf{d}$ und $\mathbf{e}$ finden, treten bei Bis(amino)stannylenen des Strukturtyps 1 dann in Lösung auf, wenn die tert-Butylgruppen an den Stickstoffatomen $z$. B. durch Isopropylgruppen ersetzt sind. Durch Reaktion mit dem stark 
basischen Pyridin kann man diese tricyclischen Systeme zu monocyclischen PyridinAddukten abbauen ${ }^{4)}$. Das Dimere $\mathbf{2 b}$ wird demgegenüber durch dieselbe Base nicht angegriffen, wie Reaktionsgleichung (12a) lehrt. Das Zielmolekül 3 muß daher über alternative Methoden dargestellt werden, was uns durch die Umsetzungen (12b) und (12c) gelungen ist.

Reaktion (12b) ist analog zu der Synthese (9a), während bei (12c) aus 4 b und 2 Äquivalenten Pyridin neben 3 das Addukt von Pyridin an Trimethylaluminium resultiert. Nach ${ }^{1}$ H-NMR-Spektrum besitzt 3 eine Spiegelebene: neben einem Resonanzsignal für die Methylgruppe am Aluminiumatom, zwei Signalen für die Methylgruppen am Silicium und einem Multiplett für die Wasserstoffatome des Pyridins findet man ein einziges Singulett für die beiden tert-Butylgruppen.

\section{Experimenteller Teil}

Alle Reaktionen wurden unter Stickstoff als Schutzgas in Stockschen Vakuumapparaturen durchgeführt. Die verschiedenen Darstellungsmethoden sind mit Buchstaben bezeichnet, die sich jeweils auf die Gleichungen im allgemeinen Teil beziehen.

${ }^{1}$ H-NMR-Spektren: Hitachi-Perkin-Elmer-Gerät (R-24B, $60 \mathrm{MHz}$ ), - NMR-Spektren anderer Kerne: Jeol-Multikern Gerät (FX, $100 \mathrm{MHz}$ ). - IR-Spektren: Perkin-Elmer $137 \mathrm{G}$ und Beckman IR 4260. - Massenspektren: Varian-MAT-CH 7 (GBF, Stöckheim). - Die Kristallparameter wurden an Weissenberg- und Buerger-Kameras bestimmt. - C,H,N-Analysen führte die Firma Beller, Göttingen, durch. Alle anderen quantitativen Elementbestimmungen erfolgten auf naßchemischem Wege.

Dimeres des 1,3-Di-tert-butyl-2,2,4-trimethyl-1,3,2,4 $\lambda^{3}$-diazasilaaluminetidins (2b), des $-1,3,2,4 \lambda^{3}$-diazasilaindetidins (2d) und des 1,3-Di-tert-butyl-2,2-dimethyl-4-phenyl-1,3,2,4 $\lambda^{3}$ diazasilaaluminetidins (2e)

Methode a): Auf $4.79 \mathrm{~g}$ (15 mmol) 1,3-Di-tert-butyl-2,2-dimethyl-1,3,2,4 $\lambda^{2}$-diazasilastannetidin (1b) ${ }^{11)}$ in $5 \mathrm{ml}$ Benzol werden $10 \mathrm{mmol}$ Trimethylaluminium ${ }^{12)}$, Triphenylaluminium ${ }^{13)}$ bzw. Trimethylindium ${ }^{14)}$ in $5 \mathrm{ml}$ Benzol zügig getropft. Anschließend wird $10 \mathrm{~min}\left(\mathrm{Al}_{2} \mathrm{Me}_{6}\right.$ und $\left.\mathrm{Al}_{2} \mathrm{Ph}_{6}\right)$ bzw. $3 \mathrm{~h}\left(\mathrm{InMe}_{3}\right)$ unter Rückfluß erhitzt. Im Fall von $\mathrm{Al}_{2} \mathrm{Me}_{6}$ bzw. $\mathrm{Al}_{2} \mathrm{Ph}_{6}$ wachsen aus der Reaktionslösung Kristalle von $\mathbf{2 b}$ bzw. $2 \mathbf{e}$, die nach Umkristallisieren aus Benzol als farblose Nadeln, (2b: $2.18 \mathrm{~g} \mathrm{(90 \% ),} \mathrm{Schmp.} 180^{\circ} \mathrm{C} ; 2 \mathrm{e}: 0.85 \mathrm{~g}(28 \%)$, Zers. $>250^{\circ} \mathrm{C}$ ) anfallen.

Bei der Umsetzung mit InMe $\mathrm{H}_{3}$ muß zunächst von einem Niederschlag, der über röntgenographische Pulvermethoden als metallisches Indium erkannt wird, abfiltriert werden. Nach Abziehen des Lösungsmittels und des überschüssigen $1 \mathrm{~b}$ i. Hochvak. sublimieren bei $110-120^{\circ} \mathrm{C} /$ 0.001 Torr $2 \mathrm{~d}$ und 4d als Gemisch. Zur Trennung wird in Benzol gelöst: $0.793 \mathrm{~g}(24 \%) 2 \mathrm{~d}$ kristallisieren aus (Schmp. $165^{\circ} \mathrm{C}$ ), während $\mathbf{4 d}$ in Losung verbleibt. Die bei allen Reaktionen entstehenden polymeren Dimethylstannane bleiben in der Reaktionslösung gelöst und können über bekannte Methoden 15.16) nachgewiesen werden.

Methode b): $3.0 \mathrm{mmol} 4 \mathrm{~b}$ bzw. $4 \mathrm{~d}$ in $3 \mathrm{ml}$ Benzol werden unter ständigem Rühren mit $0.96 \mathrm{~g}$ (3.0 mmol) 1b in $1 \mathrm{ml} \mathrm{Benzol} \mathrm{innerhalb} \mathrm{von} 5 \mathrm{~min}$ tropfenweise versetzt. Die Lösung färbt sich durch Bildung der Dimethylstannane ${ }^{15,16)}$ augenblicklich tiefrot. Nach 10min. Aufkochen unter Rückfluß und nach langsamem Abkühlen kristallisieren $0.53 \mathrm{~g}(73 \%) 2 \mathrm{~b}$ bzw. $0.48 \mathrm{~g}$ (48\%) $2 \mathrm{~d}$.

Methode c): $2.56 \mathrm{~g}(8.0 \mathrm{mmol}) 4 \mathrm{~b}$ in $25 \mathrm{ml}$ Benzol werden mit $2.8 \mathrm{~g}(8.0 \mathrm{mmol})$ 1,3-Di-tertbutyl-2,2,4,4-tetramethyl-1,3,2,4 $\lambda^{4}$-diazasilastannetidin (7), das vorsichtig zugegeben wird, 15 min unter Rückfluß erhitzt. Nach Einengen der Lösung auf die Hälfte kristallisieren $3.53 \mathrm{~g}$

Chem. Ber. 118 (1985) 
$(91 \%) 2$ b. Tetramethylstannan verbleibt in Lösung und kann IR- und NMR-spektroskopisch charakterisiert werden.

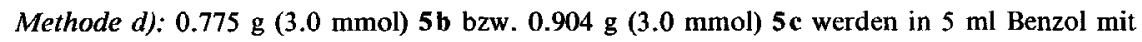
$3.0 \mathrm{mmol}$ Butyllithium (Hexanlösung, $1.6 \mathrm{M}$ ) tropfenweise versetzt Nach 0.5 h Kochen unter Rückfluß werden $0.6 \mathrm{~g}(3.0 \mathrm{mmol})$ Chlortrimethylstannan in $1 \mathrm{ml}$ Benzol zugefügt, nochmals wird kurz zum Sieden erhitzt und vom ausgefallenen Lithiumchlorid abfiltriert. Beim Einengen der Lösung kristallisieren $0.63 \mathrm{~g}(87 \%) \mathbf{~ b}$. Im Fall der Reaktion mit $5 \mathrm{c}$ erhält man die erwartete Menge Tetramethylstannan bei der Destillation $(0.5 \mathrm{~g}, 93 \%)$. Der verbleibende feste Rückstand sublimiert bei $60^{\circ} \mathrm{C} / 0.001$ Torr und stellt nach dem ${ }^{1} \mathrm{H}$-NMR-Spektrum ein Substanzgemisch dar, das sich nicht weiter trennen läßt.

Methode e): $0.42 \mathrm{~g}$ (1.63 mmol) $5 \mathrm{~b}$ werden in $3 \mathrm{ml} \mathrm{Xylol} 10 \mathrm{~h}$ unter Rückfluß erhitzt. Nach Einengen der Lösung fallen $0.115 \mathrm{~g} \mathrm{(29 \% )}$ klare, farblose Kristalle von $\mathbf{2 b}$ aus.

2b: ${ }^{1} \mathrm{H}-\mathrm{NMR}$ (Benzol): $\delta=-0.03\left(\mathrm{~s} ; 6 \mathrm{H}, \mathrm{AlCH}_{3}\right), 0.48\left(\mathrm{~s} ; 6 \mathrm{H}, \mathrm{SiCH}_{3}\right), 0.58\left(\mathrm{~s} ; 6 \mathrm{H}, \mathrm{SiCH}_{3}\right)$, 1.25 (s; $\left.18 \mathrm{H}, \mathrm{CCH}_{3}\right), 1.50$ (s; $18 \mathrm{H}, \mathrm{CCH}_{3}$ ). - MS (70 eV, hochauflösend): Ber. für $\mathrm{C}_{22} \mathrm{H}_{54}{ }^{27} \mathrm{Al}_{2} \mathrm{~N}_{4}{ }^{28} \mathrm{Si}_{2}\left(\mathrm{M}^{+}\right)$484.3518, gef. $m / e=484.3519$. - Röntgenographische Einkristalluntersuchung: Monoklin, $P 2_{1} / c ; a=1120(3), b=865(2), c=1735(4) \mathrm{pm}, \beta=118.5(1)^{\circ}$; $V=1477 \cdot 10^{6} \mathrm{pm}^{3} ; Z=2$.

$$
\begin{array}{ll}
\mathrm{C}_{22} \mathrm{H}_{54} \mathrm{Al}_{2} \mathrm{~N}_{4} \mathrm{Si}_{2} \text { (484.8) } & \text { Ber. C 54.50 H 11.23 Al 11.14 N } 11.56 \\
& \text { Gef. C 52.82 H 11.29 Al 10.83 N 10.13 } \\
& \text { Molmasse 492.5 (kryoskop. in Benzol) }
\end{array}
$$

2d: 'H-NMR (Benzol): $\delta=0.47\left(\mathrm{~s} ; 6 \mathrm{H}, \mathrm{InCH}_{3}\right.$ ), $0.48\left(\mathrm{~s} ; 12 \mathrm{H}, \mathrm{SiCH}_{3}\right), 1.33$ (s; $36 \mathrm{H}$, $\left.\mathrm{CCH}_{3}\right)$. - MS (70 eV): $m / e=330\left(\mathrm{M}^{+}, \mathrm{C}_{11} \mathrm{H}_{27}{ }^{115} \mathrm{InN}_{2}{ }^{28} \mathrm{Si}\right.$, monomer). - Röntgenographische Einkristalluntersuchung: Monoklin, $P 2_{1} / c ; a=1132.3(4), b=868.8(3), c=1794.4(8) \mathrm{pm}$, $\beta=117.4(1)^{\circ} ; V=1567 \cdot 10^{6} \mathrm{pm}^{3} ; Z=2$.

$\mathrm{C}_{22} \mathrm{H}_{54} \mathrm{In}_{2} \mathrm{~N}_{4} \mathrm{Si}_{2}(660.5) \quad$ Ber. C $40.01 \mathrm{H} 8.24$

Gef. C 38.87 H 8.28 Molmasse 674 (kryoskop. in Benzol)

2e: ${ }^{1} \mathrm{H}-\mathrm{NMR}$ (Benzol): $\delta=0.31\left(\mathrm{~s} ; 6 \mathrm{H}, \mathrm{SiCH}_{3}\right), 0.66\left(\mathrm{~s} ; 6 \mathrm{H}, \mathrm{SiCH}_{3}\right), 1.08\left(\mathrm{~s} ; 18 \mathrm{H}, \mathrm{CCH}_{3}\right)$, $1.80\left(\mathrm{~s} ; 18 \mathrm{H}, \mathrm{CCH}_{3}\right), \mathrm{m}$ im aromat. Bereich verdeckt.

$\mathrm{C}_{32} \mathrm{H}_{58} \mathrm{Al}_{2} \mathrm{~N}_{4} \mathrm{Si}_{2}$ (609.0) Ber. C 63.11 H $9.60 \mathrm{~N} 9.20$

Gef. C 61.52 H 9.11 N 8.95 Molmasse 614 (kryoskop. in Benzol)

Pyridin-Addukt von 1,3-Di-tert-butyl-2,2,4-trimethyl-1,3,2,4 $\lambda^{3}$-diazasilaaluminetidin (3)

Methode a): $0.727 \mathrm{~g}(3.0 \mathrm{mmol}) 2 \mathrm{~b}$ werden mit $0.248 \mathrm{ml}(3.0 \mathrm{mmol})$ frisch destilliertem Pyridin in $3 \mathrm{ml}$ Toluol umgesetzt. Bei $-78^{\circ} \mathrm{C}$ kristallisiert unverändert $2 \mathrm{~b}$ aus. Auch das ${ }^{1} \mathrm{H}-\mathrm{NMR}$ Spektrum der Lösung zeigt keine Reaktion zwischen $\mathbf{2 b}$ und Pyridin an!

Methode b): $0.759 \mathrm{~g}(5.0 \mathrm{mmol})$ des Addukts von Pyridin an Trimethylaluminium, das man rein durch Sublimation des Reaktionsproduktes aus $\mathrm{Al}_{2} \mathrm{Me}_{6}$ und Pyridin bei $22^{\circ} \mathrm{C} / 0.001$ Torr erhält, werden in $4 \mathrm{ml}$ Toluol langsam auf eine Lösung von $1.60 \mathrm{~g}(5.0 \mathrm{mmol}) 1 \mathrm{~b}$ in $5 \mathrm{ml}$ Toluol getropft. Eine Rotfärbung zeigt die Bildung von Dimethylstannanen an 15,16). Nach Ende des Zutropfens wird das Lösungsmittel abgezogen und dann der Rückstand durch zweimalige Sublimation bei $75^{\circ} \mathrm{C} / 0.001$ Torr gereinigt. Ausb. $1.09 \mathrm{~g} \mathrm{(68 \% )} \mathrm{3,} \mathrm{Schmp.} 67^{\circ} \mathrm{C}$.

Methode c): Zu $0.324 \mathrm{~g}$ (1.03 mmol) $4 \mathrm{~b}$ in $2 \mathrm{ml}$ Benzol gibt man $0.17 \mathrm{~g}$ (2.06 mmol) Pyridin, wobei sich die Lösung gelb färbt. Nach Abkondensieren des Lösungsmittels verbleibt ein gelbes Öl, aus dem bei $22^{\circ} \mathrm{C} / 0.001$ Torr $0.14 \mathrm{~g}(72 \%)$ farbloses, kristallines $\mathrm{Me}_{3} \mathrm{Al} \cdot \mathrm{NC}_{5} \mathrm{H}_{5}$ sublimiert, das über eine Referenzprobe (siehe oben) als solches erkannt wird. In einer zweiten Fraktion erhält man bei $75^{\circ} \mathrm{C} / 0.001$ Torr einen gelben, wachsartigen Feststoff, aus dem durch wiederholte Sublimation $0.245 \mathrm{~g}(74 \%)$ reines 3 gewonnen wird (Schmp. $\left.67^{\circ} \mathrm{C}\right) .-{ }^{1} \mathrm{H}-\mathrm{NMR}$ (Benzol): $\delta=$ 
$-0.25\left(\mathrm{~s} ; 3 \mathrm{H}, \mathrm{AlCH}_{3}\right), 0.44\left(\mathrm{~s}\right.$, breit; $\left.6 \mathrm{H}, \mathrm{SiCH}_{3}\right), 1.10\left(\mathrm{~s} ; 18 \mathrm{H}, \mathrm{CCH}_{3}\right), 7.4(\mathrm{~m} ; 5 \mathrm{H}$, aromat. $\mathrm{H}$, in Hexan als Lösungsmittel). $-{ }^{1} \mathrm{H}-\mathrm{NMR}\left(\left[\mathrm{D}_{8}\right] \mathrm{Toluol},-43^{\circ} \mathrm{C}\right): \delta=0.12\left(\mathrm{~s} ; 3 \mathrm{H}, \mathrm{AlCH}_{3}\right), 0.57$ (s; $\left.3 \mathrm{H}, \mathrm{SiCH}_{3}\right), 0.64\left(\mathrm{~s} ; 3 \mathrm{H}, \mathrm{SiCH}_{3}\right), 1.22\left(\mathrm{~s} ; 18 \mathrm{H}, \mathrm{CCH}_{3}\right)$.

$$
\begin{array}{ll}
\mathrm{C}_{16} \mathrm{H}_{32} \mathrm{AlN}_{3} \mathrm{Si} \text { (321.5) } & \text { Ber. C } 59.77 \mathrm{H} 10.03 \mathrm{Al} 8.39 \mathrm{~N} 13.07 \\
& \text { Gef. C } 58.23 \mathrm{H} 10.10 \mathrm{Al} 8.91 \mathrm{~N} 12.86 \\
& \text { Molmasse } 331.4 \text { (kryoskop. in Benzol) }
\end{array}
$$

2,4-Di-tert-butyl-1,1,3,3,5,5-hexamethyl-2,4-diaza-3-sila-1,5-dialuminapentan (4b), -1,5-digallapentan $(4 \mathrm{c})$ und -1,5-diindapentan $(4 \mathrm{~d})$

Methode a): $\mathrm{Zu}$ einer Lösung von $30 \mathrm{mmol}^{\text {Trimethylaluminium }}{ }^{12)}$, -gallium ${ }^{17)}$ bzw. -indium ${ }^{14)}$ in $20 \mathrm{ml}$ Benzol werden tropfenweise unter schnellem Rühren $1.60 \mathrm{~g} \mathrm{(5.0} \mathrm{mmol)} 1 \mathrm{~b}^{11)}$ in $5 \mathrm{ml}$ Benzol gegeben. Die orangefarbene Lösung wird $30 \mathrm{~min}$ bei Raumtemp. gerührt. Nach Abziehen des Lösungsmittels und der überschüssigen Trimethylelement-Verbindung wird der verbleibende Rückstand i.Vak. erhitzt: es sublimieren $4 \mathrm{~b}$ bei $80^{\circ} \mathrm{C} / 0.001$ Torr, $4 \mathrm{c}$ bei $86^{\circ} \mathrm{C} /$ 0.001 Torr und $4 \mathrm{~d}$ bei $95^{\circ} \mathrm{C} / 0.001$ Torr. Zur Reinigung wird die Sublimation wiederholt, wobei erhalten werden: $1.37 \mathrm{~g}(87 \%) 4 \mathrm{~b}$ mit Schmp. $180^{\circ} \mathrm{C}, 1.64 \mathrm{~g}(82 \%) 4 \mathrm{c}$ und $2.30 \mathrm{~g} \mathrm{(94 \% )} 4 \mathrm{~d}$ mit Schmp. $130^{\circ} \mathrm{C}$ (Zers.).

Methode b): $\mathrm{Zu} 1.73 \mathrm{~g}$ (12 mmol) $\mathrm{Al}_{2} \mathrm{Me}_{6}$ in $10 \mathrm{ml}$ Benzol tropft man unter schnellem Rühren eine Lösung von $2.09 \mathrm{~g}(6.0 \mathrm{mmol}) 7$ (Darstellung siehe weiter unten) in $5 \mathrm{ml}$ Benzol und erhitzt anschließend unter Rückfluß. Nach dem Abkondensieren von Lösungsmittel, überschüssigem $\mathrm{Al}_{2} \mathrm{Me}_{6}$ und Tetramethylstannan, das sich spektroskopisch charakterisieren läßt, sublimieren $1.73 \mathrm{~g}(92 \%) 4 \mathrm{~b}$ bei $80^{\circ} \mathrm{C} / 0.001$ Torr.

Methode c): $5.0 \mathrm{mmol} \mathbf{5} \mathrm{b}$ bzw. $\mathbf{5} \mathbf{d}$ werden mit äquimolaren Mengen Trimethylaluminium bzw. -indium in $5 \mathrm{ml}$ Benzol 30 min unter Rückfluß gekocht. Nach Abziehen des Lösungsmittels i. Vak. sublimieren $1.45 \mathrm{~g}(92 \%) 4 \mathrm{~b}$ bei $80^{\circ} \mathrm{C} / 0.001$ Torr bzw. $2.30 \mathrm{~g}(94 \%) 4 \mathrm{~d}$ bei $95^{\circ} \mathrm{C} / 0.001$ Torr.

Methode d): $\mathrm{Zu}$ einer Lösung von $0.28 \mathrm{ml}(1.15 \mathrm{mmol}) N, N^{\prime}$-Di-tert-butyl-Si,Si-dimethylsilandiamin (8) ${ }^{18)}$ in $5 \mathrm{ml}$ Diethylether tropft man $2.3 \mathrm{mmol}$ Butyllithium (1.6 M Hexanlösung) und kocht 2.5 h unter Rückfluß. Diese Reaktionslösung wird auf eine Aufschlämmung von $0.416 \mathrm{~g}$ Dimethylindiumchlorid ${ }^{19)}$ in $5 \mathrm{ml}$ Diethylether getropft und anschließend aufgekocht. Vom ausgefallenen Lithiumchlorid filtriert man ab und entfernt destillativ das Lösungsmittel. Nach zweimaliger Sublimation bei $95^{\circ} \mathrm{C} / 0.001$ Torr erhält man $0.39 \mathrm{~g}(69 \%) 4 \mathrm{~d}$.

$4 \mathrm{~b}$ : IR (Benzol): $2950 \mathrm{vs,} 2920 \mathrm{sh}, 2880 \mathrm{sh}, 2855 \mathrm{sh}, 2818 \mathrm{sh}, 1455 \mathrm{w}, 1383 \mathrm{w}, 1361 \mathrm{~s}, 1258 \mathrm{sh}$, $1250 \mathrm{~m}, 1226 \mathrm{~m}, 1192 \mathrm{vs}, 1078 \mathrm{w} / \mathrm{br}, 989 \mathrm{vs}, 945 \mathrm{w}, 855 \mathrm{~s}, 812 \mathrm{~s}, 791 \mathrm{~s}, 752 \mathrm{~s}, 712 \mathrm{~cm}^{-1} \mathrm{sh}$. ${ }^{1} \mathrm{H}$-NMR (Benzol, $\left.30^{\circ} \mathrm{C}\right): \delta=-0.18\left(\mathrm{~s} ; 12 \mathrm{H}, \mathrm{AlCH}_{3}\right), 0.47\left(\mathrm{~s} ; 6 \mathrm{H}, \mathrm{SiCH}_{3}\right), 1.08(\mathrm{~s} ; 18 \mathrm{H}$, $\mathrm{CCH}_{3}$ ). $\quad{ }^{13} \mathrm{C}-\mathrm{NMR}\left(\left[\mathrm{D}_{6}\right]\right.$ Benzol, entkoppelt): $\delta=-4.0$ (s, breit; $\mathrm{AlCH}_{3}$ ), 8.38 (s; $\mathrm{SiCH}_{3}$ ), $34.21\left(\mathrm{~s} ; \mathrm{C}-\mathrm{CH}_{3}\right), 51.68\left(\mathrm{~s} ; \mathrm{N}-\mathrm{C}-\mathrm{CH}_{3}\right) .-{ }^{14} \mathrm{~N}-\mathrm{NMR}\left(\left[\mathrm{D}_{6}\right]\right.$ Benzol, ext. $\left.\mathrm{NH}_{4}^{+}\right): \delta=90(\mathrm{~s}$, breit; $\left.w_{1 / 2}=340 \mathrm{~Hz}\right) .-{ }^{27} \mathrm{Al}-\mathrm{NMR}\left(\left[\mathrm{D}_{6}\right]\right.$ Benzol, ext. $\left.\mathrm{Al}^{3+}\right): \delta=159$ (s, breit; $w_{1 / 2}=82.3 \mathrm{~Hz}$ ). - MS (70 eV): Peak höchster Masse: $m / e=299\left(\mathrm{M}-\mathrm{CH}_{3}\right)$. - Gitterkonstanten der Elementarzelle siehe Tab. 1.

$$
\begin{aligned}
\mathrm{C}_{14} \mathrm{H}_{36} \mathrm{Al}_{2} \mathrm{~N}_{2} \mathrm{Si} \text { (314.5) } & \text { Ber. C 53.47 H 11.54 Al 17.16 N } 8.91 \\
& \text { Gef. C 53.90 H 11.38 Al 17.28 N } 8.50 \\
& \text { Molmasse 318.6 (kryoskop. in Benzol) }
\end{aligned}
$$

4c: ${ }^{1} \mathrm{H}$-NMR (Benzol, $\left.30^{\circ} \mathrm{C}\right): \delta=0.18\left(\mathrm{~s} ; 12 \mathrm{H}, \mathrm{GaCH}_{3}\right), 0.46\left(\mathrm{~s} ; 6 \mathrm{H}, \mathrm{SiCH}_{3}\right), 1.05(\mathrm{~s} ; 18 \mathrm{H}$, $\left.\mathrm{CCH}_{3}\right)$. - MS (70 eV): Zersetzung von $4 \mathrm{c}$ unter Bildung von $\mathrm{GaMe}_{3}\left(m / e=114\left(\mathrm{C}_{3} \mathrm{H}_{9}{ }^{69} \mathrm{Ga}\right)\right)$ und $2 \mathrm{c}\left(m / e=284\left(\mathrm{C}_{11} \mathrm{H}_{27}{ }^{69} \mathrm{GaN}_{2}{ }^{28} \mathrm{Si}\right)\right)$.

$$
\mathrm{C}_{14} \mathrm{H}_{36} \mathrm{Ga}_{2} \mathrm{~N}_{2} \mathrm{Si}(400.0) \text { Ber. C } 42.04 \mathrm{H} 9.07 \mathrm{Ga} 34.86 \text { N } 7.00
$$

Gef. C 40.19 H 8.64 Ga 34.32 N 6.67

Molmasse 393 (kryoskop. in Benzol) 
4d: ${ }^{1} \mathrm{H}-\mathrm{NMR}$ (Benzol, $30^{\circ} \mathrm{C}$ ): $\delta=0.17\left(\mathrm{~s} ; 12 \mathrm{H}, \mathrm{InCH}_{3}\right.$ ), $0.43\left(\mathrm{~s} ; 6 \mathrm{H}, \mathrm{SiCH}_{3}\right.$ ), $1.00(\mathrm{~s} ; 18 \mathrm{H}$, $\left.\mathrm{CCH}_{3}\right)$. - MS (70 eV): Peak höchster Masse: $m / e=475\left(\mathrm{C}_{13} \mathrm{H}_{33}{ }^{115} \mathrm{InN}_{2}{ }^{28} \mathrm{Si}\left(\mathrm{M}-\mathrm{CH}_{3}\right)\right.$ ).

$\mathrm{C}_{14} \mathrm{H}_{36} \mathrm{In}_{2} \mathrm{~N}_{2} \mathrm{Si}(490.2)$ Ber. C 34.30 H 7.40 N 5.72 Gef. C 33.82 H 7.48 N 5.38

2,4-Di-tert-butyl-1,1,5,5-tetrachlor-3,3-dimethyl-2,4-diaza-3-sila-1,5-dialuminapentan (4 e): $\mathrm{Zu}$ $0.541 \mathrm{~g}(1.72 \mathrm{mmol}) 4 \mathrm{~b}$ in $3 \mathrm{ml}$ Benzol wird eine Lösung von $1.37 \mathrm{~g} \mathrm{(6.88} \mathrm{mmol)} \mathrm{Chlortrimethyl-}$ stannan in $6 \mathrm{ml}$ Benzol getropft. Die Temperatur der Reaktionslösung sollte $35^{\circ} \mathrm{C}$ nicht überschreiten. Nach Abkondensieren des Lösungsmittels und des Tetramethylstannans sublimieren bei $80^{\circ} \mathrm{C} / 0.001$ Torr $0.45 \mathrm{~g}(66 \%)$ farblose, wachsartige Kristalle von $4 \mathrm{e}$, die sich bei $115^{\circ} \mathrm{C}$ zersetzen. - IR (Benzol): 2955 s, $2895 \mathrm{sh}, 2860 \mathrm{sh}, 1450 \mathrm{w}, 1403 \mathrm{w}, 1375 \mathrm{~m}, 1360 \mathrm{~m}, 1255 \mathrm{~m}$, $1222 \mathrm{vs}, 1165 \mathrm{~m}, 1090 \mathrm{~s}, 935 \mathrm{w}, 910 \mathrm{w}, 875 \mathrm{vs}, 821 \mathrm{~m}, 788 \mathrm{~s}, 750 \mathrm{~cm}^{-1} \mathrm{~m}$. $-{ }^{1} \mathrm{H}-\mathrm{NMR}$ (Benzol): $\delta=0.46\left(\mathrm{~s} ; 6 \mathrm{H}, \mathrm{SiCH}_{3}\right), 1.15\left(\mathrm{~s} ; 18 \mathrm{H}, \mathrm{CCH}_{3}\right)$.

$$
\begin{array}{cl}
\mathrm{C}_{10} \mathrm{H}_{24} \mathrm{Al}_{2} \mathrm{Cl}_{4} \mathrm{~N}_{2} \mathrm{Si} \text { (396.3) } & \text { Ber. C } 30.32 \mathrm{H} 6.11 \mathrm{Al} 13.62 \mathrm{Cl} 35.80 \mathrm{~N} 7.07 \\
& \text { Gef. C } 30.36 \mathrm{H} 6.24 \mathrm{Al} 13.59 \mathrm{Cl} 35.31 \mathrm{~N} 7.06 \\
& \text { Molmasse 389.2 (kryoskop. in Benzol) }
\end{array}
$$

Umsetzungen von Trimethylaluminium mit 1,3-Di-tert-butyl-2,2-dimethyl-1,3,2,4 $4 \lambda^{2}$-diazasilagermetidin (1 a) und -plumbetidin (1 c): $\left.\mathrm{Zu} 0.43 \mathrm{~g} \mathrm{(3.0} \mathrm{mmol)} \mathrm{Al}_{2} \mathrm{Me}_{6}{ }^{12}\right)$ in $3 \mathrm{ml} \mathrm{Hexan} \mathrm{tropft} \mathrm{man}$ langsam unter Rühren $0.5 \mathrm{mmol} \mathbf{1}^{20)}$ bzw. $\mathbf{1}^{20)}$, die jeweils in $2 \mathrm{ml}$ Benzol gelöst sind. Nach kurzem Erhitzen bis zur Siedetemp. der Lösungsmittel werden diese neben überschüssigem $\mathrm{Al}_{2} \mathrm{Me}_{6}$ i. Vak. abgezogen (im Fall der Reaktion mit $1 \mathrm{c}$ muß vorher noch von einem dunkelgrauen Niederschlag abfiltriert werden). Aus dem festen Rückstand gewinnt man durch Sublimation bei $80^{\circ} \mathrm{C} / 0.001$ Torr $0.75 \mathrm{~g}(79 \%) 4 \mathrm{~b}$, ausgehend von $1 \mathrm{a}$, und $0.76 \mathrm{~g}(81 \%) 4 \mathrm{~b}$, ausgehend von $1 \mathrm{c}$. $4 \mathrm{~b}$ wird spektroskopisch und durch physikalische Daten charakterisiert.

1,3-Di-tert-butyl-2,2,4,4-tetramethyl-1,3-diaza-2-sila-4-borabutan (5a), -4-aluminabutan (5b), -4-gallabutan (5c), -4-indabutan (5d) und-4-ihallabutan (5e)

Methode a): In eine Lösung von $1.83 \mathrm{~g}$ (5.0 mmol) $N, N^{\prime}$-Di-tert-butyl- $N$-(trimethylstannyl)Si,Si-dimethylsilandiamin (9) (Darstellung siehe weiter unten) in $5 \mathrm{ml}$ Benzol tropft man äquimolare Mengen der entsprechenden Trimethylelement-Verbindungen $\left.\left(\frac{1}{2} \mathrm{Al}_{2} \mathrm{Me}_{6}{ }^{12}\right), \mathrm{GaMe}_{3}{ }^{17}\right)$, $\mathrm{InMe}_{3}{ }^{14)}, \mathrm{TlMe}_{3}{ }^{21)}$ ), die in $5 \mathrm{ml}$ Benzol gelöst sind. Nach $10 \mathrm{~min}$. Rühren bei $40^{\circ} \mathrm{C}$ wird Benzol zusammen mit Tetramethylstannan abkondensiert. Der Rückstand wird destilliert. Es fallen an: $1.22 \mathrm{~g} \mathrm{(95 \% )} \mathrm{5h,} 1.41 \mathrm{~g}(94 \%) 5 \mathrm{c}, 1.64 \mathrm{~g} \mathrm{(95 \% )} \mathrm{5d} \mathrm{und} 2.02 \mathrm{~g}(93 \%) 5 \mathrm{e}$.

Methode b): $0.60 \mathrm{~g}$ (5.0 mmol) Dimethylborbromid22) in $3 \mathrm{ml}$ Benzol werden mit $1.83 \mathrm{~g}$ $(5.0 \mathrm{mmol}) 9$, das in $5 \mathrm{ml}$ Benzol gelöst ist, tropfenweise versetzt. Nach kurzem Erhitzen bis zum Sieden des Lösungsmittels wird i. Vak. Bromtrimethylstannan zusammen mit Benzol in eine Kühlfalle abgezogen. Anschließend destillieren $1.15 \mathrm{~g}(95 \%)$ der farblosen Flüssigkeit $5 \mathbf{a}$.

Methode c): $1.22 \mathrm{ml}$ (5.0 mmol) $N, N^{\prime}$-Di-tert-butyl-Si, Si-dimethylsilandiamin (8) ${ }^{18)}$ werden zusammen mit $5.0 \mathrm{mmol}$ Trimethylaluminium bzw. -gallium in $25 \mathrm{ml} p$-Xylol $1 \mathrm{~h}$ auf $130^{\circ} \mathrm{C}$ erhitzt. Nach Abziehen des Lösungsmittels sublimieren bzw. destillieren $1.12 \mathrm{~g}(87 \%) \mathbf{5 b}$ bzw. $1.11 \mathrm{~g}$ (74\%) 5c. Verwendet man zur Reaktion mit 8 Trimethylindium, so erhäit man ausschließlich $0.89 \mathrm{~g}(72 \%) 4 \mathrm{~d}$.

Methode d): $0.46 \mathrm{~g}(1.46 \mathrm{mmol}) 4 \mathrm{~b}$ werden zusammen mit $0.36 \mathrm{ml}(1.46 \mathrm{mmol}) 8^{18)}$ in $3 \mathrm{ml} \mathrm{Ben}$ zol $24 \mathrm{~h}$ unter Rückfluß erhitzt. Nach Abkondensieren des Lösungsmittels sublimieren $0.13 \mathrm{~g}$ $(35 \%) 5$ b.

Methode e): $\mathrm{Zu} 0.356 \mathrm{~g}(1.13 \mathrm{mmol}) 4 \mathrm{~b}$ in $3 \mathrm{ml}$ Benzol fügt man tropfenweise $0.12 \mathrm{ml}$ $(1.16 \mathrm{mmol})$ Diethylamin und erhitzt anschließend $5 \mathrm{~min}$ unter Rückfluß. Die flüchtigen Bestandteile lassen sich bei $25^{\circ} \mathrm{C} / 0.001$ Torr abkondensieren (u.a. die Ringverbindung $\left(\mathrm{Me}_{2} \mathrm{Al}\right)_{2}-$ $\left(\mathrm{NEt}_{2}\right)_{2}{ }^{23)}$ ). Das verbleibende $\mathrm{O} l$ wird in $1 \mathrm{ml}$ Hexan aufgenommen, woraus bei $-50^{\circ} \mathrm{C} 0.103 \mathrm{~g}$ $(35 \%)$ farblose Nadeln von $5 \mathrm{~b}$ kristallisieren. 
5a: Sdp. $42^{\circ} \mathrm{C} / 0.001$ Torr. $-{ }^{1} \mathrm{H}-\mathrm{NMR}$ (Benzol): $\delta=0.37$ (s; $6 \mathrm{H}, \mathrm{SiCH}_{3}$ ), 0.68 (s, breit; $6 \mathrm{H}$, $\left.\mathrm{BCH}_{3}\right), 1.08\left(\mathrm{~s} ; 9 \mathrm{H}, \mathrm{CCH}_{3}\right), 1.32\left(\mathrm{~s} ; 9 \mathrm{H}, \mathrm{CCH}_{3}\right)$. - MS (70 eV): Peak höchster Masse: $m / e=$ 227 , ber. für $\mathrm{C}_{11} \mathrm{H}_{28}{ }^{11} \mathrm{BN}_{2}{ }^{28} \mathrm{Si}\left(\mathrm{M}-\mathrm{CH}_{3}\right)$.

\section{$\mathrm{C}_{12} \mathrm{H}_{31} \mathrm{BN}_{2} \mathrm{Si}(242.3)$ Ber. C 59.49 H 12.90 N 11.56 Gef. C 58.35 H 12.33 N 11.48}

5 b: Schmp. $45^{\circ} \mathrm{C}$, Subl.-P. $40^{\circ} \mathrm{C} / 0.001$ Torr. - IR (Benzol): $3224 \mathrm{w}, 2950 \mathrm{vs,} 2805 \mathrm{w} / \mathrm{sh}$, $1448 \mathrm{w}, 1395 \mathrm{w}, 1375 \mathrm{~m}, 1355 \mathrm{~m}, 1250 \mathrm{~m}, 1230 \mathrm{vs}, 1200 \mathrm{~s}, 1185 \mathrm{~s}, 1095 \mathrm{~s}, 1005 \mathrm{w}, 950 \mathrm{~m}, 916 \mathrm{~m}$, $897 \mathrm{~m}, 862 \mathrm{vs}, 815 \mathrm{vs}, 781 \mathrm{sh}, 770 \mathrm{~s}, 755 \mathrm{~m}, 695 \mathrm{~cm}^{-1} \mathrm{w} .-{ }^{1} \mathrm{H}-\mathrm{NMR}$ (Benzol, $30^{\circ} \mathrm{C}$ ): $\delta=-0.38$ (s; $3 \mathrm{H}, \mathrm{AlCH}_{3}$ ), $-0.26\left(\mathrm{~s} ; 3 \mathrm{H}, \mathrm{AlCH}_{3}\right), 0.14$ (s; $3 \mathrm{H}, \mathrm{SiCH}_{3}$ ), 0.30 (s; $3 \mathrm{H}, \mathrm{SiCH}_{3}$ ), 0.91 (s; $9 \mathrm{H}$, $\mathrm{CCH}_{3}$ ), 1.22 (s; $\left.9 \mathrm{H}, \mathrm{CCH}_{3}\right) .-{ }^{13} \mathrm{C}-\mathrm{NMR}$ ( $\left[\mathrm{D}_{8}\right]$ Toluol, entkoppelt, Stand. Toluol): $\delta=-4.7$ (breit; $\left.\mathrm{AlCH}_{3}\right), 6.07\left(\mathrm{~s} ; \mathrm{SiCH}_{3}\right), 6.41\left(\mathrm{~s} ; \mathrm{SiCH}_{3}\right), 31.22\left(\mathrm{~s} ; \mathrm{C}-\mathrm{CH}_{3}\right), 35.51\left(\mathrm{~s} ; \mathrm{C}-\mathrm{CH}_{3}\right), 49.84$ (s; $\left.\mathrm{N}-\mathrm{C}-\mathrm{CH}_{3}\right), 52.71\left(\mathrm{~s} ; \mathrm{N}-\mathrm{C}-\mathrm{CH}_{3}\right) .-{ }^{14} \mathrm{~N}-\mathrm{NMR}\left(\left[\mathrm{D}_{8}\right]\right.$ Toluol, ext. $\left.\mathrm{NH}_{4}^{+}\right): \delta=84$ (s, breit; $\left.w_{1 / 2}=540 \mathrm{~Hz}\right) .-{ }^{15} \mathrm{~N}-\mathrm{NMR}\left(\left[\mathrm{D}_{8}\right]\right.$ Toluol, ext. $\left.\mathrm{NH}_{3}\right): \delta=73.4(\mathrm{~s}) . \quad{ }^{29} \mathrm{Si}-\mathrm{NMR}\left(\left[\mathrm{D}_{8}\right]\right.$ Toluol; ext. TMS): $\delta=0.99(\mathrm{~s}) .-{ }^{27} \mathrm{Al}-\mathrm{NMR}\left(\left[\mathrm{D}_{8}\right]\right.$ Toluol; ext. Al $\left.{ }^{3+}\right): \delta=159$ (breit; $w_{1 / 2}=2800 \mathrm{~Hz}$ ). - MS (70 eV): Peak höchster Masse: $m / e=243\left(\mathrm{M}-\mathrm{CH}_{3}\right)$.

$$
\begin{array}{ll}
\mathrm{C}_{12} \mathrm{H}_{31} \mathrm{AlN}_{2} \mathrm{Si} \text { (258.5) } & \text { Ber. C } 55.77 \text { H } 12.09 \text { Al 10.44 N } 10.84 \\
& \text { Gef. C } 55.31 \text { H } 12.34 \text { Al 10.38 N 10.01 } \\
& \text { Molmasse 264.7 (kryoskop. in Benzol) }
\end{array}
$$

5c: Schmp. $18^{\circ} \mathrm{C}$, Sdp. $48^{\circ} \mathrm{C} / 0.001$ Torr. $-{ }^{1} \mathrm{H}$-NMR (Benzol, $30^{\circ} \mathrm{C}$ ): $\delta=0.01$ (breit; $6 \mathrm{H}$, $\left.\mathrm{GaCH}_{3}\right), 0.24$ (breit; $\left.6 \mathrm{H}, \mathrm{SiCH}_{3}\right), 0.89\left(\mathrm{~s} ; 9 \mathrm{H}, \mathrm{CCH}_{3}\right), 1.16\left(\mathrm{~s} ; 9 \mathrm{H}, \mathrm{CCH}_{3}\right) .-\mathrm{MS}(70 \mathrm{eV})$ : Peak höchster Masse und Intensität: $m / e=285\left(\mathrm{C}_{11} \mathrm{H}_{28}{ }^{69} \mathrm{GaN}_{2}{ }^{28} \mathrm{Si}, \mathrm{M}-\mathrm{CH}_{3}\right)$.

$$
\begin{array}{lllll}
\mathrm{C}_{12} \mathrm{H}_{31} \mathrm{GaN}_{2} \mathrm{Si}(301.2) & \text { Ber. C } 47.85 & \mathrm{H} 10.37 & \mathrm{Ga} 23.15 & \mathrm{~N} 9.30 \\
& \text { Gef. C } 49.02 & \mathrm{H} 10.28 & \mathrm{Ga} 23.41 & \mathrm{~N} 9.06
\end{array}
$$

5d: Schmp. $20^{\circ} \mathrm{C}$, Sdp. $68^{\circ} \mathrm{C} / 0.001$ Torr. $-{ }^{1} \mathrm{H}-\mathrm{NMR}$ (Benzol, $\left.30^{\circ} \mathrm{C}\right): \delta=0.05(\mathrm{~s} ; 6 \mathrm{H}$, $\left.\mathrm{InCH}_{3}\right), 0.25\left(\mathrm{~s} ; 6 \mathrm{H}, \mathrm{SiCH}_{3}\right), 0.88\left(\mathrm{~s} ; 9 \mathrm{H}, \mathrm{CCH}_{3}\right), 1.12\left(\mathrm{~s} ; 9 \mathrm{H}, \mathrm{CCH}_{3}\right)$. - MS (70 eV): Peak höchster Masse: $m / e=331\left(\mathrm{C}_{11} \mathrm{H}_{28}{ }^{115} \mathrm{InN}_{2}{ }^{28} \mathrm{Si}, \mathrm{M}-\mathrm{CH}_{3}\right)$.

$\mathrm{C}_{12} \mathrm{H}_{31} \mathrm{InN}_{2} \mathrm{Si}$ (346.3) Ber. C $41.62 \mathrm{H} 9.02 \mathrm{~N} 8.09$

Gef. C 43.03 H 9.07 N 8.33 Molmasse 355 (kryoskop. in Cyclohexan)

5e: Sdp. $74^{\circ} \mathrm{C} / 0.001$ Torr. $-{ }^{1} \mathrm{H}-\mathrm{NMR}\left(\right.$ Benzol, $\left.30^{\circ} \mathrm{C}\right): \delta=0.76\left(2 \mathrm{~d},{ }^{2} J\left({ }^{203} \mathrm{Tl}-{ }^{1} \mathrm{H}\right)=44 \mathrm{~Hz}\right.$, $\left.{ }^{2} J\left({ }^{205} \mathrm{Tl}-{ }^{1} \mathrm{H}\right)=337 \mathrm{~Hz} ; 6 \mathrm{H}, \mathrm{TlCH}_{3}\right), 0.28\left(\mathrm{~s} ; 6 \mathrm{H}, \mathrm{SiCH}_{3}\right), 0.90\left(\mathrm{~s} ; 9 \mathrm{H}, \mathrm{CCH}_{3}\right), 1.18(\mathrm{~s} ; 9 \mathrm{H}$, $\left.\mathrm{CCH}_{3}\right)$. - MS (70 eV): Zersetzung.

\section{$\mathrm{C}_{12} \mathrm{H}_{31} \mathrm{~N}_{2} \mathrm{SiTl}(435.9) \quad$ Ber. C 33.07 H 7.17 N 6.43 Gef. C 34.55 H 7.41 N 6.75}

1,3-Di-tert-butyl-4,4-dichlor-2,2-dimethyl-1,3-diaza-2-sila-4-borabutan (5f): $\mathrm{Zu}$ einer Lösung von $0.68 \mathrm{~g}(5.8 \mathrm{mmol})$ Bortrichlorid 21$)$ in $2 \mathrm{ml}$ Toluol tropft man bei $-70^{\circ} \mathrm{C} 2.12 \mathrm{~g}(5.8 \mathrm{mmol})$ 1,3-Di-tert-butyl-2,2,4,4-tetramethyl-1,3-diaza-2-sila-4-stannapentan (9, Vorschrift siehe weiter unten), das in $3 \mathrm{ml}$ Toluol gelöst ist. Nach Erwärmen auf Raumtemp. werden alle flüchtigen Bestandteile bei 0.001 Torr abgezogen. Aus dem Rückstand destillieren $0.57 \mathrm{~g}(35 \%) 5 \mathrm{f}$ als klare Flüssigkeit, Sdp. $53^{\circ} \mathrm{C} / 0.001$ Torr. $-{ }^{1} \mathrm{H}-\mathrm{NMR}$ (Benzol): $\delta=0.51\left(\mathrm{~s} ; 6 \mathrm{H}, \mathrm{SiCH}_{3}\right), 1.14(\mathrm{~s} ; 9 \mathrm{H}$, $\left.\mathrm{CCH}_{3}\right), 1.31$ (s; $\left.9 \mathrm{H}, \mathrm{CCH}_{3}\right)$. - MS $(70 \mathrm{eV})$ : Peak höchster Masse: $m / e=286\left(\mathrm{C}_{10} \mathrm{H}_{25}{ }^{11} \mathrm{~B}-\right.$ $\left.{ }^{37} \mathrm{Cl}_{2} \mathrm{~N}_{2} \mathrm{Si}, \mathrm{M}\right)$.

$$
\begin{array}{rlllll}
\mathrm{C}_{10} \mathrm{H}_{25} \mathrm{BCl}_{2} \mathrm{~N}_{2} \mathrm{Si}(283.1) & \text { Ber. } \mathrm{C} 42.42 & \mathrm{H} 8.90 & \mathrm{Cl} 25.04 & \mathrm{~N} & 9.89 \\
& \text { Gef. C } 42.50 & \mathrm{H} 8.63 & \mathrm{Cl} 23.91 & \mathrm{~N} 10.23
\end{array}
$$

I,3-Di-tert-butyl-4,4-dichlor-2,2-dimethyl-1,3-diaza-2-sila-4-aluminabutan (5g): In eine Lösung von $0.596 \mathrm{~g}$ ( $1.99 \mathrm{mmol}) 5 \mathrm{~b}$ in $5 \mathrm{ml}$ Benzol tropft man unter Rühren $0.793 \mathrm{~g}$ (3.98 mmol) Chlortrimethylstannan, das in $3 \mathrm{ml}$ Benzol gelöst ist. Danach erhitzt man kurz zum Sieden und kondensiert anschließend Benzol und entstandenes Tetramethylstannan ab. Bei der Sublimation des farblosen Rückstandes gewinnt man $0.453 \mathrm{~g} \mathrm{(76 \% )} 5 \mathrm{~g}$. Schmp. $70^{\circ} \mathrm{C}$, Subl.-P. $60^{\circ} \mathrm{C} /$ 0.001 Torr. $-{ }^{1} \mathrm{H}-\mathrm{NMR}\left(\mathrm{Benzol}, 30^{\circ} \mathrm{C}\right.$ ): $\delta=0.07$ (s; $3 \mathrm{H}, \mathrm{SiCH}_{3}$ ), 0.23 (s; $3 \mathrm{H}, \mathrm{SiCH}_{3}$ ), 1.01 (s; 
$\left.9 \mathrm{H}, \mathrm{CCH}_{3}\right), 1.24\left(\mathrm{~s} ; 9 \mathrm{H}, \mathrm{CCH}_{3}\right)$ - MS $(70 \mathrm{eV})$ : Peak höchster Masse: $m / e=283\left(\mathrm{C}_{9} \mathrm{H}_{22} \mathrm{Al}-\right.$ ${ }^{35} \mathrm{Cl}_{2} \mathrm{~N}_{2}{ }^{28} \mathrm{Si}, \mathrm{M}-\mathrm{CH}_{3}$ ).

$$
\begin{array}{lllll}
\mathrm{C}_{10} \mathrm{H}_{25} \mathrm{AlCl}_{2} \mathrm{~N}_{2} \mathrm{Si} \text { (299.3) } & \text { Ber. C } 40.13 \mathrm{H} 8.42 \mathrm{Cl} 23.69 \mathrm{~N} 9.36 \\
& \text { Gef. C } 41.19 \mathrm{H} 8.36 \mathrm{Cl} 26.32 \text { N } 9.53
\end{array}
$$

1,3-Di-tert-butyl-4,4-dichlor-2,2-dimethyl-1,3-diaza-2-sila-4-gallabutan (5h) und -4-indabutan (5i): $\left.\mathrm{Zu} 2.02 \mathrm{~g}(10.0 \mathrm{mmol}) 8^{18}\right)$ in $60 \mathrm{ml}$ Diethylether gibt man unter Rühren äquimolare Mengen an Butyllithium und erhitzt $2 \mathrm{~h}$ unter Rückfluß. Die erhaltene Lösung wird auf eine Aufschlämmung von 11.0 mmol Gallium-24) bzw. Indiumtrichlorid 24) in $60 \mathrm{ml}$ Diethylether getropft. Nach $10 \mathrm{~h}$ Erhitzen wird vom farblosen Niederschlag abfiltriert und das Lösungsmittel abkondensiert. Der feste Rückstand wird durch Sublimation gereinigt. Ausb. $2.67 \mathrm{~g}(78 \%) 5 \mathrm{~h}$ und $1.55 \mathrm{~g} \mathrm{(40 \% )}$ $5 \mathbf{i}$.

5h: Schmp. $56^{\circ} \mathrm{C}$, Subl.-P. $55^{\circ} \mathrm{C} / 0.001$ Torr. $-{ }^{1} \mathrm{H}-\mathrm{NMR}$ (Benzol, $30^{\circ} \mathrm{C}$ ): $\delta=0.05$ (s, breit; $\left.3 \mathrm{H}, \mathrm{SiCH}_{3}\right), 0.19$ (s, breit; $\left.3 \mathrm{H}, \mathrm{SiCH}_{3}\right), 0.96\left(\mathrm{~s} ; 9 \mathrm{H}, \mathrm{CCH}_{3}\right), 1.20\left(\mathrm{~s} ; 9 \mathrm{H}, \mathrm{CCH}_{3}\right) .-\mathrm{MS}(70 \mathrm{eV})$ : Peak höchster Masse: $m / e=329\left(\mathrm{C}_{9} \mathrm{H}_{22}{ }^{37} \mathrm{Cl}_{2}{ }^{69} \mathrm{GaN}_{2}{ }^{28} \mathrm{Si}, \mathrm{M}-\mathrm{CH}_{3}\right)$.

$$
\begin{array}{llllll}
\mathrm{C}_{10} \mathrm{H}_{25} \mathrm{Cl}_{2} \mathrm{GaN}_{2} \mathrm{Si}(342.0) & \text { Ber. C } 35.12 \mathrm{H} 7.37 \mathrm{Cl} 20.73 \mathrm{~N} 8.19 \\
& \text { Gef. C } 36.18 \mathrm{H} 7.61 \mathrm{Cl} 19.55 \text { N } 8.14
\end{array}
$$

5i: Schmp. $61^{\circ} \mathrm{C}$, Subl.-P. $70^{\circ} \mathrm{C} / 0.001$ Torr. $-{ }^{1} \mathrm{H}-\mathrm{NMR}$ (Benzol, $30^{\circ} \mathrm{C}$ ): $\delta=0.12$ (s, breit; $\left.6 \mathrm{H}, \mathrm{SiCH}_{3}\right), 0.92\left(\mathrm{~s} ; 9 \mathrm{H}, \mathrm{CCH}_{3}\right), 1.12\left(\mathrm{~s} ; 9 \mathrm{H}, \mathrm{CCH}_{3}\right) .-\mathrm{MS}(70 \mathrm{eV})$ : Peak höchster Masse: $m / e=370\left(\mathrm{C}_{9} \mathrm{H}_{21}{ }^{35} \mathrm{Cl}_{2}{ }^{115} \mathrm{InN}_{2}{ }^{28} \mathrm{Si}, \mathrm{M}-\mathrm{CH}_{4}\right)$.

$$
\begin{array}{llllllll}
\mathrm{C}_{10} \mathrm{H}_{25} \mathrm{Cl}_{2} \mathrm{InN}_{2} \mathrm{Si} \text { (387.1) } & \text { Ber. } \mathrm{C} 31.03 & \mathrm{H} 6.51 & \mathrm{Cl} 18.32 & \mathrm{~N} 7.24 & \mathrm{Si} 7.25 \\
& \text { Gef. } \mathrm{C} 31.19 & \mathrm{H} 6.63 & \mathrm{Cl} 17.76 & \mathrm{~N} 7.32 & \mathrm{Si} 7.25
\end{array}
$$

1,3-Di-tert-butyl-2,2,4,4-tetramethyl-1-oxa-3-aza-2-sila-4-aluminabutan (6a): Zu einer Lösung von $2 \mathrm{ml}(8.1 \mathrm{mmol})$ tert-Butoxy(tert-butylamino)dimethylsilan (10) $\left.{ }^{25}\right)$ in $3 \mathrm{ml}$ Benzol tropft man langsam $3.4 \mathrm{ml}$ einer Hexanlösung von Trimethylaluminium $\left(0.6 \mathrm{~g}(4.13 \mathrm{mmol}) \mathrm{Al}_{2} \mathrm{Me}_{6}\right)$. Es tritt sofort Gasentwicklung auf (Methan), wobei sich die Lösung auf $40^{\circ} \mathrm{C}$ erwärmt. Nach dem Abkondensieren der Lösungsmittel verbleibt eine klare, farblose Flüssigkeit, aus der bei $110^{\circ} \mathrm{C} /$ 12 Torr $1.773 \mathrm{~g}(84 \%) 6$ a destilliert, das bei $-4^{\circ} \mathrm{C}$ fest wird. - IR (Benzol): $2950 \mathrm{~s}, 2890 \mathrm{sh}$, $2860 \mathrm{sh}, 2810 \mathrm{sh}, 1445 \mathrm{w}, 1392 \mathrm{~m}, 1355 \mathrm{~m}, 1254 \mathrm{~s}, 1232 \mathrm{~s}, 1180 \mathrm{~s} / \mathrm{br}, 1106 \mathrm{~m}, 935 \mathrm{~s}, 870 \mathrm{~s}, 817 \mathrm{~s}$, $777 \mathrm{~cm}^{-1}$ s. $-{ }^{1} \mathrm{H}-\mathrm{NMR}(\mathrm{Benzol}): \delta=-0.37\left(\mathrm{~s} ; 6 \mathrm{H}, \mathrm{AlCH}_{3}\right), 0.24\left(\mathrm{~s} ; 6 \mathrm{H}, \mathrm{SiCH}_{3}\right), 1.07(\mathrm{~s} ; 9 \mathrm{H}$, $\left.\mathrm{CCH}_{3}\right), 1.21\left(\mathrm{~s} ; 9 \mathrm{H}, \mathrm{CCH}_{3}\right)$.

$$
\begin{array}{ll}
\mathrm{C}_{12} \mathrm{H}_{30} \mathrm{AINOSi} \text { (259.5) } & \text { Ber. C 55.55 H } 11.66 \mathrm{Al} 10.40 \mathrm{~N} 5.40 \\
& \text { Gef. C 53.86 H } 11.37 \mathrm{Al} 10.12 \mathrm{~N} 4.82 \\
& \text { Molmasse } 266.7 \text { (kryoskop. in Benzol) }
\end{array}
$$

1,3-Di-tert-butyl-4,4-dichlor-2,2-dimethyl-1-oxa-3-aza-2-sila-4-gallabutan (6b): $\mathrm{Zu} 8.53 \mathrm{~g} \mathrm{(42}$ mmol) 1025) in $50 \mathrm{ml} \mathrm{Hexan} \mathrm{werden} 42 \mathrm{mmol}$ tert-Butyllithium (1.6 M Hexanlösung) getropft und danach $2 \mathrm{~h}$ gekocht. Unter Eiskühlung versetzt man die Lösung dann tropfenweise mit einer Lö-

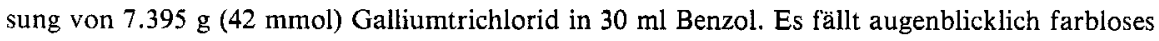
Lithiumchlorid aus. Nach $4 \mathrm{~h}$ Rühren bei $60^{\circ} \mathrm{C}$ wird vom Niederschlag abfiltriert und das Lösungsmittel unter reduziertem Druck abgezogen. Mittels Sublimation bei $50^{\circ} \mathrm{C} / 0.001$ Torr erhält man $12.1 \mathrm{~g} \mathrm{(84 \% )} 6 \mathrm{~b}$ als farbloses Pulver. $-{ }^{1} \mathrm{H}-\mathrm{NMR}$ (Benzol): $\delta=0.16\left(\mathrm{~s} ; 6 \mathrm{H}, \mathrm{SiCH}_{3}\right), 0.97$ (s; $\left.9 \mathrm{H}, \mathrm{CCH}_{3}\right), 0.99$ (s; $\left.9 \mathrm{H}, \mathrm{CCH}_{3}\right)$. - MS (70 eV): Zersetzung.

$$
\begin{aligned}
\mathrm{C}_{10} \mathrm{H}_{24} \mathrm{Cl}_{2} \text { GaNOSi (343.0) } & \text { Ber, C } 35.02 \mathrm{H} 7.05 \mathrm{Ga} 20.33 \mathrm{~N} 4.08 \\
& \text { Gef. C } 34.59 \mathrm{H} 7.13 \mathrm{Ga} 19.75 \mathrm{~N} 4.04 \\
& \text { Molmasse } 338 \text { (kryoskop. in Benzol) }
\end{aligned}
$$

1,3-Di-tert-butyl-2,2,4,4-tetramethyl-1,3,2,4 $\lambda^{4}$-diazasilastannetidin (7): $3.8 \mathrm{~g}$ (18.77 mmol) $N, N^{\prime}$-Di-tert-butyl-Si,Si-dimethylsilandiamin (8) ${ }^{18)}$ werden unter Rühren mit einer Lösung von $37.54 \mathrm{mmol} n$-Butyllithium in $23.5 \mathrm{ml}$ Hexan versetzt. Nach $1 \mathrm{~h}$ Kochen gibt man eine Lösung von 
$3.45 \mathrm{~g}$ (15.71 mmol) Dichlordimethylstannan in $5 \mathrm{ml}$ Benzol hinzu und erhitzt abermals $1 \mathrm{~h}$ unter Rückfluß. Vom ausgefallenen Lithiumchlorid wird abfiltriert und das Filtrat fraktionierend destilliert. Bei $100^{\circ} \mathrm{C} / 0.001$ Torr gewinnt man $3.78 \mathrm{~g}(69 \%)$ einer farblosen Flüssigkeit, die bei $25^{\circ} \mathrm{C}$ fest wird. Sie wird als 7 charakterisiert. $-{ }^{1} \mathrm{H}-\mathrm{NMR}$ (Benzol): $\delta=0.41$ (s, Superposition, erkenntlich an der relativen Intensität der ${ }^{117 / 119} \mathrm{Sn}$-Satelliten; $12 \mathrm{H}, \mathrm{SiCH}_{3}$ und $\mathrm{SnCH}_{3}$, ${ }^{2} J\left({ }^{1} \mathrm{H}^{117 / 119} \mathrm{Sn}\right)=58.5$ bzw. 56.2), $1.11\left(\mathrm{~s} ; 18 \mathrm{H}, \mathrm{CCH}_{3}\right)$.

$$
\begin{array}{lllll}
\mathrm{C}_{12} \mathrm{H}_{30} \mathrm{~N}_{2} \mathrm{SiSn}(349.2) & \text { Ber. C } 41.28 \text { H } 8.66 \text { N } 8.02 \text { Sn } 33.99 \\
& \text { Gef. C } 41.68 \text { H } 8.65 \text { N } 7.90 \text { Sn } 33.21
\end{array}
$$

1,3-Di-tert-butyl-2,2,4,4-tetramethyl-1,3-diaza-2-sila-4-stannapentan (9): $0.95 \mathrm{ml}$ (3.9 mmol) $8{ }^{18)}$ in $5 \mathrm{ml}$ Hexan werden mit $1.85 \mathrm{mmol}$ Butyllithium, das in $1.2 \mathrm{ml} \mathrm{Hexan} \mathrm{gelöst} \mathrm{ist,} \mathrm{versetzt}$ und $1.5 \mathrm{~h}$ bei $50^{\circ} \mathrm{C}$ gehalten. Diese Lösung fügt man anschließend tropfenweise zu einer Lösung von $0.37 \mathrm{~g}(1.85 \mathrm{mmol})$ Chlortrimethylstannan in $5 \mathrm{ml}$ Hexan und rührt abermals $2 \mathrm{~h}$ bei $50^{\circ} \mathrm{C}$. Nach Abfiltrieren vom ausgefallenen Lithiumchlorid wird die Lösung i. Vak, eingeengt. Bei $80^{\circ} \mathrm{C} / 0.001$ Torr destillieren $0.54 \mathrm{~g}(80 \%)$ einer klaren Flüssigkeit, die als 9 charakterisiert wird. - IR (Benzol): $3280 \mathrm{~cm}^{-1}(\mathrm{v}(\mathrm{N}-\mathrm{H}))$. $-{ }^{1} \mathrm{H}-\mathrm{NMR}$ (Benzol): $\delta=0.27\left(\mathrm{~s} ; 6 \mathrm{H}, \mathrm{SiCH}_{3}\right.$ ), 0.35 (s und $\left.\mathrm{d}, 9 \mathrm{H}, \mathrm{SnCH}_{3},{ }^{2} J\left({ }^{1} \mathrm{H}^{-117 / 119} \mathrm{Sn}\right)=56 \mathrm{bzw} .58 \mathrm{~Hz}\right), 1.18\left(\mathrm{~s} ; 9 \mathrm{H}, \mathrm{CCH}_{3}\right), 1.33(\mathrm{~s} ; 9 \mathrm{H}$, $\left.\mathrm{CCH}_{3}\right)$. - MS (70 eV): Peak höchster Masse: $m / e=351\left(\mathrm{C}_{12} \mathrm{H}_{31} \mathrm{~N}_{2}{ }^{28} \mathrm{Si}^{120} \mathrm{Sn}, \mathrm{M}-\mathrm{CH}_{3}\right)$.

$\mathrm{C}_{13} \mathrm{H}_{34} \mathrm{~N}_{2} \mathrm{SiSn}(365.2)$ Ber. C 42.75 H 9.38 N 7.67 Gef. C 43.96 H 9.79 N 7.73

1) Weitere in Arbeit befindliche Veröffentlichungen tragen die Untertitel: II. Strukturelle Untersuchungen und III. Dynamische Phänomene und Lewis-Säure-Stärke.

2) M. Veith, Nachr. Chem. Tech. Lab. 30, 940 (1982).

3) $M$. Veith und O. Recktenwald, Top. Curr. Chem. 104, 1 (1982).

4) $M$. Veith, Z. Naturforsch., Teil B 33, 1 (1978).

5) W. Storch, W. Jackstiess, H. Nöth und G. Winter, Angew. Chem. 89, 494 (1977); Angew. Chem., Int. Ed. Engl. 16, 478 (1977).

6) C. Grugel, W. P. Neumann und M. Schriewer, Angew. Chem. 91, 577 (1979); Angew. Chem., Int. Ed. Engl. 18, 543 (1979).

7) P. G. Harrison und J. J. Zuckerman, J. Am. Chem. Soc. 92, 2577 (1970).

8) M. F. Lappert, P. P. Power, A. R. Sanger und R. C. Srivastava, Metal and Metalloid Amides, E. Horwood, Chichester, England 1980.

9) H. G. Grimm, Z. Elektrochem. 31, 474 (1925).

10) M. Veith, Z. Naturforsch., Teil B 33, 7 (1978).

11) M. Veith, Angew. Chem. 87, 287 (1975); Angew. Chem., Int. Ed. Engl. 14, 263 (1975).

12) S. Pasynkiewicz und M. Boleslawski, J. Organomet. Chem. 25, 29 (1970).

13) W. Menzel, Z. Anorg. Allg. Chem. 269, 52 (1952).

14) H. C. Clark und A. L. Pickard, J. Organomet. Chem. 8, 427 (1967).

15) $W$. P. Neumann, The Stannylenes in M. Gielen und $P$. G. Harrison, The Organomet. and Coord. Chem. of Ge, $\mathrm{Sn}$ and $\mathrm{Pb}$, Freund Publ., Tel Aviv 1978.

16) Th. L. Brown und G. L. Morgan, Inorg. Chem. 2, 736 (1963).

17) E. Wiberg, T. Johannsen und O. Stecher, Z. Anorg. Allg. Chem. 251, 114 (1943).

18) W. Fink, Helv. Chim. Acta 47, 498 (1964).

19) K. Yasuda und R. Okawara, Inorg. Nucl. Chem. Lett. 3, 135 (1967).

20) $M$. Veith und $M$. Grosser, Z. Naturforsch., Teil B 37, 1375 (1982).

21) G. Brauer, Handbuch der Präp. Anorg. Chemie, S. $797\left(\mathrm{BCl}_{3}\right)$ und S. $874\left(\mathrm{TlMe}_{3}\right)$, F. Enke Verlag, Stuttgart 1978.

22) W. Schabacher und J. Goubeau, Z. Anorg. Allg. Chem. 294, 183 (1958).

23) H. C. Brown und N. Davidson, J. Am. Chem. Soc. 64, 316 (1942).

24) H. Hecht, G. Jander und H. Schlapmann, Z. Anorg. Allg. Chem. 254, 255 (1947).

25) $M$. Veith und $R$. Rösler, J. Organomet. Chem. 229, 131 (1982).

26) W. J. Dunning, Phys. Chem. Solids 18, 21 (1961).

27) I. Haiduc, The Chemistry of Inorganic Ring Systems, S. 1018-1061, Wiley, New York 1970.

28) S. Cucinella, T. Salvatori, C. Busetto und A. Mazzei, J. Organomet. Chem. 108, 13 (1976). 